\title{
Stochastic Stabilization of Itô Stochastic Systems with Markov Jumping and Linear Fractional Uncertainty
}

\author{
Fei Long, ${ }^{1,2}$ Hongmei Huang, ${ }^{1,2}$ and Adan Ding ${ }^{1,2}$ \\ ${ }^{1}$ Institute of Intelligent Information Processing, Guizhou University, Guiyang 550025, China \\ ${ }^{2}$ College of Computer Science and Information, Guizhou University, Guiyang 550025, China \\ Correspondence should be addressed to Fei Long; flong1973@yahoo.com.cn
}

Received 17 September 2012; Revised 19 December 2012; Accepted 6 January 2013

Academic Editor: Derong Liu

Copyright (c) 2013 Fei Long et al. This is an open access article distributed under the Creative Commons Attribution License, which permits unrestricted use, distribution, and reproduction in any medium, provided the original work is properly cited.

\begin{abstract}
For a class of Itô stochastic linear systems with the Markov jumping and linear fractional uncertainty, the stochastic stabilization problem is investigated via state feedback and dynamic output feedback, respectively. In order to guarantee the stochastic stability of such uncertain systems, state feedback and dynamic output control law are, respectively, designed by using multiple Lyapunov function technique and LMI approach. Finally, two numerical examples are presented to illustrate our results.
\end{abstract}

\section{Introduction}

Modeled by a set of linear systems with the transitions among the linear systems governed by the Markov chain, the Markov jumping linear system (MJLS) can characterize and model different types of systems [1], for instance, fault-tolerant systems, target tracking systems, manufactory processes, networked control systems, multiagent systems, and so on. In the past years, many important results on MJLS have been addressed in the literature, such as, the stability analysis and control design which were discussed in [2-8]. Besides the aforementioned theoretical studies, MJLS also found applications in practical systems, such as networked direct current motor systems [9].

The interest of this topic lies in the fact that many practical systems, with random abrupt changes, sudden environmental changes, and so on, can be modeled by Itô stochastic linear systems with Markov jumping and configurable uncertainty [10]. Therefore, it is very important to investigate robustly stochastic stabilization problem for the aforementioned Itô stochastic MJLS with linear fractional uncertainties. Recently, some results for Itô stochastic MJLS have been addressed in [11-18].

In this paper, motivated by the recent work [19], we consider robustly stochastic stabilization problem for Markov jumping Itô stochastic linear system subject to linear fractional uncertainty. An effective LMI-based approach for state feedback and dynamic output feedback controller is designed such that the closed-loop system is robust stable in the mean square sense, respectively.

The reminder of this paper is organized as follows: the problem statement is described in Section 2, while in Section 3 the stochastic stability for Itô stochastic MJLS with linear fractional uncertainty is addressed. In Section 4, the robustly stochastic stabilization of Itô stochastic MJLS with linear fractional uncertainty is investigated via state feedback and dynamical output feedback, respectively. Two numerical examples are presented in Section 5 to illustrate the obtained results. Finally, some conclusions are drawn in Section 6.

\section{Problem Formulation}

Consider the following unforced Itô stochastic MJLS with linear fractional uncertainties:

$$
\begin{gathered}
d x(t)=A(t, r(t)) x(t) d t+W(r(t)) x(t) d \omega(t), \\
x(0)=x_{0}, \quad r(0)=r_{0},
\end{gathered}
$$

where $\{r(t), t \geq 0\}$ is a Markov process taking values in a finite space $\overline{\mathbb{N}}=\{1,2, \ldots, N\}$ and $x(t) \in \mathbb{R}^{n}$ is the state vector. $\omega(t) \in \mathbb{R}$ is a standard Wiener process that is supposed to be independent of the Markov process $\{r(t), t \geq 0\} . x_{0} \in \mathbb{R}^{n}$ denotes the initial state of system at $t=0$, and $r_{0} \in \overline{\mathbb{N}}$ stands for the initial mode in the Markov process at $t=0$. 
$A(t, r(t))$ and $W(r(t)), r(t)=i \in \overline{\mathbb{N}}$ are system matrices with compatible dimensions that depend on $r(t)$ and $t$, and

$$
\begin{gathered}
A(t, i)=A(i)+M_{A}(i) H_{A}(t, i) N_{A}(i), \\
H_{A}(t, i)=\Sigma_{A}(t, i)\left[I-G_{A}(i) \Sigma_{A}(t, i)\right]^{-1},
\end{gathered}
$$

where $A(i), M_{A}(i), N_{A}(i)$, and $G_{A}(i)$ are known real constant matrices with appropriate dimensions. $\Sigma_{A}(t, i)$ is an unknown real matrix function with Lebesgue-measurable elements, satisfying $\Sigma_{A}^{T}(t, i) \Sigma_{A}(t, i) \leq I$ with $I$ being the identity matrix.

The evolution of the Markov process $\{r(t), t \geq 0\}$ is governed by the following probability transitions:

$$
\begin{aligned}
P & {[r(t+h)=j \mid r(t)=i] } \\
& = \begin{cases}\lambda_{i j} h+o(h), & r(t) \text { jumps from mode } i \text { to mode } j, \\
1+\lambda_{i i} h+o(h), & r(t) \text { stays at mode } i,\end{cases}
\end{aligned}
$$

where $\lambda_{i j} \geq 0$ is the transition rate from mode $i$ to mode $j$ at time instant $t$ when $i \neq j$, and $\lambda_{i i}=-\sum_{j=1, j \neq i}^{N} \lambda_{i j} \cdot o(h)$ is the little- $o$ notation defined by $\lim _{h \rightarrow 0}(o(h) / h)=0$.

For the stochastic stability, we adopt the following definition. For more details, please refer to [18] and the references therein.

Definition 1. The Itô stochastic MJLS in (1) with all modes and all $t \geq 0$ is said to achieve stochastic stability if there exists a finite positive constant $T\left(x_{0}, r_{0}\right)$ such that the following inequality holds for any initial condition $\left(x_{0}, r_{0}\right)$ :

$$
E\left[\int_{0}^{\infty}\|x(t)\|^{2} d t \mid\left(x_{0}, r_{0}\right)\right] \leq T\left(x_{0}, r_{0}\right)
$$

where $E[\cdot \mid \cdot]$ is the expectation conditioning on the initial value of $\left(x_{0}, r_{0}\right)$.

\section{Stochastic Stability}

Before proceeding further, we recall the following lemma, which will be used in the proof of the stochastic stability of the Itô stochastic MJLS in (1).

Lemma 2 (see [20]). Let $H, E$ be given constant matrices with appropriate dimensions and norm-bounded time-varying uncertain matrix $\Sigma(t)$ with $\Sigma^{T}(t) \Sigma(t) \leq I$. For any $\varepsilon>0$, one has

$$
H \Sigma(t) E+E^{T} \Sigma^{T}(t) H^{T} \leq \varepsilon H H^{T}+\varepsilon^{-1} E^{T} E .
$$

Lemma 3 (see [19]). Let the matrix $G$ satisfy $G^{T} G<I$; define the following set:

$$
\xi=\left\{H(t)=\Sigma(t)[I-G \Sigma(t)]^{-1}, \Sigma^{T}(t) \Sigma(t) \leq I\right\} .
$$

Then, the set $\xi$ can be rewritten as

$$
\begin{gathered}
\xi=\left\{H(t)=\left(I-G^{T} G\right)^{-1} G^{T}+\left(I-G^{T} G\right)^{-1 / 2} \Pi(t) ;\right. \\
\left.\Pi^{T}(t) \Pi(t) \leq I+G\left(I-G^{T} G\right)^{-1} G^{T}\right\} .
\end{gathered}
$$

Theorem 4. The Itô stochastic uncertain MJLS in (1) is stochastically stable if there exists a set of symmetric and positive-definite matrices $\{P(i) \mid i=1,2, \ldots, N\}$ and $a$ set of positive scalars $\left\{\varepsilon_{A}(i) \mid i=1,2, \ldots, N\right\}$ such that the following sets of coupled inequalities hold for each $i \in \overline{\mathbb{N}}$ and all admissible linear fractional uncertainties:

$$
\left(\begin{array}{cccc}
J_{0}(i) & * & * & * \\
R_{A 0}^{T}(i) & -Q_{A}(i) & 0 & 0 \\
\varepsilon_{A}(i) N_{A}(i) & 0 & -I & 0 \\
P(i) W(i) & 0 & 0 & -P(i)
\end{array}\right)<0
$$

where $*$ represents the blocks that are induced by symmetry and

$$
\begin{gathered}
J_{0}(i)=A^{T}(i) P(i)+P(i) A(i)+\sum_{j=1}^{N} \lambda_{i j} P(j), \\
Q_{A}(i)=I-G_{A}^{T}(i) G_{A}(i), \\
R_{A 0}(i)=\varepsilon_{A}(i) N_{A}^{T}(i) G_{A}(i)+\varepsilon_{A}^{-1}(i) P(i) M_{A}(i) .
\end{gathered}
$$

Proof. Consider the following Lyapunov-like function:

$$
V(x(t), r(t))=x^{T}(t) P(r(t)) x(t),
$$

where $P(r(t))$ denotes the positive symmetric matrix. The infinitesimal generator $\zeta$ can be considered as a derivative of the Lyapunov function $V(x(t), r(t))$ along the trajectory of the Markov process $\{r(t), t \geq 0\}$ in point $(x(t), r(t))$ at time $t$. We need to derive the infinitesimal generator $\zeta$ of $V(x(t), r(t))$ first of all. According to the definition of $\zeta$, we have

$$
\begin{gathered}
\zeta V(x(t), r(t)) \\
=\lim _{\Delta t \rightarrow 0} \frac{1}{\Delta t}\{E[V(x(t+\Delta t), r(t+\Delta t)) \mid(x(t), r(t))] \\
\quad-V(x(t), r(t))\},
\end{gathered}
$$

where $\Delta t$ is a small positive number. Setting $r(t)=i$ and applying the law of total probability and the 
probability transition of the Markov process $\{r(t), t \geq 0\}$ yield

$$
\begin{gathered}
\zeta V(x(t), i) \\
\begin{array}{l}
\lim _{\Delta t \rightarrow 0} \frac{1}{\Delta t}\left[\sum_{j=1, j \neq i}^{N} \operatorname{Pr}\{r(t+\Delta t)=j \mid r(t)=i\}\right. \\
\times V(x(t+\Delta t), j)] \\
+\lim _{\Delta t \rightarrow 0} \frac{1}{\Delta t}[\operatorname{Pr}\{r(t+\Delta t)=i \mid r(t)=i\} \\
\times V(x(t+\Delta t), i)-V(x(t), i)] \\
=\lim _{\Delta t \rightarrow 0}\left[\sum_{j=1}^{N} \lambda_{i j} V(x(t+\Delta t), j)\right. \\
\left.+\frac{V(x(t+\Delta t), i)-V(x(t), i)}{\Delta t}\right] .
\end{array}
\end{gathered}
$$

According to the Itô stochastic differential coefficient theorem [18], we have

$$
\begin{aligned}
\zeta V(x(t), i)= & \sum_{j=1}^{N} \lambda_{i j} V(x(t), j)+[A(t, i) x(t)]^{T} \frac{\partial V(x, i)}{\partial x} \\
& +\frac{1}{2} \operatorname{tr}\left[x^{T}(t) W^{T}(i) \frac{\partial^{2} V(x, i)}{\partial x^{2}} W(i) x(t)\right] .
\end{aligned}
$$

Note that $\partial V(x, i) / \partial x=2 P(i) x(t)$ and $\partial^{2} V(x, i) / \partial x^{2}=2 P(i)$, then the infinitesimal generator $\zeta$ becomes

$$
\zeta V(x(t), i)=x^{T}(t) \Omega(t, i) x(t),
$$

where $\Omega(t, i)=A^{T}(t, i) P(i)+P(i) A(t, i)+\sum_{j=1}^{N} \lambda_{i j} P(j)+$ $W^{T}(i) P(i) W(i)$.

Using Lemmas 2 and 3, we have

$$
\begin{gathered}
N_{A}^{T}(i) H_{A}^{T}(t, i) M_{A}^{T}(i) P(i)+P(i) M_{A}(i) H_{A}(t, i) N_{A}(i) \\
=N_{A}^{T}(i)\left[\left(I-G_{A}^{T}(i) G_{A}(i)\right)^{-1} G_{A}^{T}(i)\right. \\
\left.\quad+\left(I-G_{A}^{T}(i) G_{A}(i)\right)^{-1 / 2} \Pi_{A}(t, i)\right]^{T} \\
\times M_{A}^{T}(i) P(i)+P(i) M_{A}(i)
\end{gathered}
$$

$$
\begin{aligned}
& \times\left[\left(I-G_{A}^{T}(i) G_{A}(i)\right)^{-1} G_{A}^{T}(i)\right. \\
& \left.+\left(I-G_{A}^{T}(i) G_{A}(i)\right)^{-1 / 2} \Pi_{A}(t, i)\right] N_{A}(i) \\
\leq & N_{A}^{T}(i) G_{A}(i) Q_{A}^{-1}(i) M_{A}^{T}(i) P(i) \\
& +P(i) M_{A}(i) Q_{A}^{-1}(i) G_{A}^{T}(i) N_{A}(i) \\
& +\varepsilon_{A}^{2}(i) N_{A}^{T}(i)\left[I+G_{A}(i) Q_{A}^{-1}(i) G_{A}^{T}(i)\right] N_{A}(i) \\
& +\varepsilon_{A}^{-2}(i) P(i) M_{A}(i) Q_{A}^{-1}(i) M_{A}^{T}(i) P(i) \\
= & R_{A 0}(i) Q_{A}^{-1}(i) R_{A 0}^{T}(i)+\varepsilon_{A}^{2}(i) N_{A}^{T}(i) N_{A}(i),
\end{aligned}
$$

where $R_{A 0}(i)=\varepsilon_{A}(i) N_{A}^{T}(i) G_{A}(i)+\varepsilon_{A}^{-1}(i) P(i) M_{A}(i), Q_{A}(i)=$ $I-G_{A}^{T}(i) G_{A}(i)$.

Hence $\Omega(t, i) \leq \widehat{\Omega}(i)$, where

$$
\begin{aligned}
& \widehat{\Omega}(i)= J_{0}(i)+R_{A 0}(i) Q_{A}^{-1}(i) R_{A 0}^{T}(i) \\
&+\varepsilon_{A}^{2}(i) N_{A}^{T}(i) N_{A}(i)+W^{T}(i) P(i) W(i), \\
& J_{0}(i)=A^{T}(i) P(i)+P(i) A(i)+\sum_{j=1}^{N} \lambda_{i j} P(j) .
\end{aligned}
$$

According to the Schur complement, $\widehat{\Omega}(i)<0, i \in \overline{\mathbb{N}}$ implies

$$
\left(\begin{array}{cccc}
J_{0}(i) & * & * & * \\
R_{A 0}^{T}(i) & -Q_{A}(i) & 0 & 0 \\
\varepsilon_{A}(i) N_{A}(i) & 0 & -I & 0 \\
P(i) W(i) & 0 & 0 & -P(i)
\end{array}\right)<0 .
$$

Therefore $(x(t), i) \quad \leq \quad x^{T}(t) \hat{\Omega}(i) x(t) \leq$ $\max _{i \in \overline{\mathbb{N}}}\left\{\lambda_{\max } \widehat{\Omega}(i)\right\} x^{T}(t) x(t)$.

By the generalized Dynkin formula, we have

$$
\begin{aligned}
E & {\left[V(x(t), i) \mid\left(x_{0}, r_{0}\right)\right]-V\left(x_{0}, r_{0}\right) } \\
& =E\left[\int_{0}^{t} \zeta V(x(s), i) d s \mid\left(x_{0}, r_{0}\right)\right] \\
& \leq \max _{i \in \overline{\mathbb{N}}}\left\{\lambda_{\max } \widehat{\Omega}(i)\right\} E\left[\int_{0}^{t} x^{T}(s) x(s) d s \mid\left(x_{0}, r_{0}\right)\right] .
\end{aligned}
$$

The last inequality implies

$$
\begin{aligned}
& -\max _{i \in \mathbb{N}}\left\{\lambda_{\max } \widehat{\Omega}(i)\right\} E\left[\int_{0}^{t} x^{T}(s) x(s) d s \mid\left(x_{0}, r_{0}\right)\right] \\
& \quad \leq V\left(x_{0}, r_{0}\right)-E\left[V(x(t), i) \mid\left(x_{0}, r_{0}\right)\right] \\
& \quad \leq V\left(x_{0}, r_{0}\right) .
\end{aligned}
$$


Furthermore, the condition in (8) indicates $\max _{i \in \overline{\mathbb{N}}}\left\{\lambda_{\text {max }} \widehat{\Omega}(i)\right\}<0$, so

$$
\begin{aligned}
& E\left[\int_{0}^{t} x^{T}(s) x(s) d s \mid\left(x_{0}, r_{0}\right)\right] \\
& \quad \leq-\frac{V\left(x_{0}, r_{0}\right)}{\max _{i \in \overline{\mathbb{N}}}\left\{\lambda_{\max } \bar{\Omega}(i)\right\}} \stackrel{\text { def }}{=} T\left(x_{0}, r_{0}\right)
\end{aligned}
$$

holds for any $t>0$. Letting $t$ go to infinity, then, we know that

$$
E\left[\int_{0}^{\infty} x^{T}(s) x(s) d s \mid\left(x_{0}, r_{0}\right)\right] \leq T\left(x_{0}, r_{0}\right) .
$$

According to Definition 1, the Itô stochastic uncertain MJLS in (1) is stochastically stable. This ends the proof.

\section{Stochastic Stabilization}

4.1. State Feedback Stochastic Stabilization. In this subsection, we discuss how to design the robust state feedback control law for the following Itô stochastic uncertain MJLS:

$$
\begin{gathered}
d x(t)=A(t, r(t)) x(t) d t+B(t, r(t)) u(t) d t \\
+W(r(t)) x(t) d \omega(t), \\
x(0)=x_{0}, \quad r(0)=r_{0},
\end{gathered}
$$

where $B(t, r(t)), r(t)=i \in \overline{\mathbb{N}}$ are system matrices with compatible dimensions that depend on $r(t)$ and $t$, and

$$
\begin{gathered}
B(t, i)=B(i)+M_{B}(i) H_{B}(t, i) N_{B}(i), \\
H_{B}(t, i)=\Sigma_{B}(t, i)\left[I-G_{B}(i) \Sigma_{B}(t, i)\right]^{-1},
\end{gathered}
$$

where $B(i), M_{B}(i), N_{B}(i)$, and $G_{B}(i)$ are known real constant matrices with appropriate dimensions. $\Sigma_{B}(t, i)$ is an unknown real matrix function with Lebesgue-measurable elements, satisfying $\Sigma_{B}^{T}(t, i) \Sigma_{B}(t, i) \leq I$ with $I$ being the identity matrix. The robust state feedback control law to be designed is

$$
u(t)=K(r(t)) x(t)
$$

where $K(r(t))$ is a gain with appropriate dimension to be determined for each mode $r(t) \in \overline{\mathbb{N}}$. Notice that the gain of the above controller is model dependent, which requires the knowledge of the model $r(t)$ at time $t$ to choose the appropriate gain among $\{K(1), K(2), \ldots, K(N)\}$. Thus when the model switches from mode $i$, which uses the gain $K(i)$, to mode $j$, the controller gains must be switched instantaneously to the gain $K(j)$ to guarantee the desired performances.

Theorem 5. Suppose that there exists a set of symmetric and positive-definite matrices $\{X(i) \mid i=1,2, \ldots, N\}$, a set of matrices $\{Y(i) \mid i=1,2, \ldots, N\}$, and a set of positive scalars $\left\{\varepsilon_{A}(i) \mid i=1,2, \ldots, N\right\}$ and $\left\{\varepsilon_{B}(i) \mid i=1,2, \ldots, N\right\}$ such that the following sets of coupled inequalities hold for each $i \in$ $\overline{\mathbb{N}}$ and all admissible linear fractional uncertainties. Then the controller (24) with gain $K(i)=Y(i) X^{-1}(i)$ can stabilize stochastically the Itô stochastic uncertain MJLS in (22)

$$
\left[\begin{array}{ccccccc}
J_{u}(i) & * & * & * & * & * & * \\
R_{A 1}^{T}(i) & -Q_{A}(i) & 0 & 0 & 0 & 0 & 0 \\
R_{B 1}^{T}(i) & 0 & -Q_{B}(i) & 0 & 0 & 0 & 0 \\
\varepsilon_{A}^{-1}(i) N_{A}(i) X(i) & 0 & 0 & -I & 0 & 0 & 0 \\
\varepsilon_{B}^{-1}(i) N_{B}(i) Y(i) & 0 & 0 & 0 & -I & 0 & 0 \\
W(i) X(i) & 0 & 0 & 0 & 0 & -X(i) & 0 \\
\mathbb{S}^{T}(X(i)) & 0 & 0 & 0 & 0 & 0 & -\mathbb{X}(i)
\end{array}\right]
$$

$<0$

where * represents the blocks that are induced by symmetry and

$$
\begin{gathered}
J_{u}(i)=X(i) A^{T}(i)+A(i) X(i)+Y^{T}(i) B^{T}(i) \\
+B(i) Y(i)+\lambda_{i i} X(i), \\
R_{A 1}(i)=\varepsilon_{A}(i) M_{A}(i)+\varepsilon_{A}^{-1}(i) X(i) N_{A}^{T}(i) G_{A}(i), \\
R_{B 1}(i)=\varepsilon_{B}(i) M_{B}(i)+\varepsilon_{B}^{-1}(i) Y^{T}(i) N_{B}^{T}(i) G_{B}(i), \\
\mathbb{X}(i)=\operatorname{diag}\{X(1), \ldots, X(i-1), X(i+1), \ldots, X(N)\}, \\
\left.\mathbb{S}(X(i)) \quad{ }^{X}, \sqrt{\lambda_{i, i-1}} I, \sqrt{\lambda_{i, i+1}} I, \ldots, \sqrt{\lambda_{i N} I}\right], \\
=X(i)\left[\sqrt{\lambda_{i 1} I, \ldots}(i)=I-G_{A}^{T}(i) G_{A}(i),\right. \\
Q_{B}(i)=I-G_{B}^{T}(i) G_{B}(i) .
\end{gathered}
$$

Proof. With the use of the state feedback control law in (24) for system (22), the closed-loop system becomes

$$
\begin{aligned}
d x(t)= & (A(t, r(t))+B(t, r(t)) K(r(t))) x(t) d t \\
& +W(r(t)) x(t) d \omega(t) .
\end{aligned}
$$

In order to guarantee the stochastic stability of system (27), using the stochastically stable condition in Theorem 4, we know that the following inequality should be satisfied for each $i \in \overline{\mathbb{N}}$ :

$$
\begin{aligned}
& {[A(t, i)+B(t, i) K(i)]^{T} P(i)+P(i)[A(t, i)+B(t, i) K(i)]} \\
& +W^{T}(i) P(i) W(i)+\sum_{j=1}^{N} \lambda_{i j} P(j)<0 .
\end{aligned}
$$

Let $X(i)=P^{-1}(i)$, and before and after multiplying the matrix inequality $(28)$ by $X(i)$, then, we have

$$
\begin{aligned}
& X(i) A^{T}(t, i)+A(t, i) X(i)+X(i) K^{T}(i) B^{T}(t, i) \\
& +B(t, i) K(i) X(i)+X(i) W^{T}(i) X^{-1}(i) W(i) X(i) \\
& +\sum_{j=1}^{N} \lambda_{i j} X(i) X^{-1}(j) X(i)<0 .
\end{aligned}
$$


Applying expressions (2) and (23) of the linear fractional uncertainties, the above inequality can be described as

$$
\begin{aligned}
X(i) & A^{T}(i)+A(i) X(i)+X(i) K^{T}(i) B^{T}(i) \\
& +B(i) K(i) X(i) \\
& +X(i) N_{A}^{T}(i) H_{A}^{T}(t, i) M_{A}^{T}(i) \\
& +M_{A}(i) H_{A}(t, i) N_{A}(i) X(i) \\
& +X(i) K^{T}(i) N_{B}^{T}(i) H_{B}^{T}(t, i) M_{B}^{T}(i) \\
& +M_{B}(i) H_{B}(t, i) N_{B}(i) K(i) X(i) \\
& +X(i) W^{T}(i) X^{-1}(i) W(i) X(i) \\
& +X(i)\left[\sum_{j=1}^{N} \lambda_{i j} X^{-1}(j)\right] X(i)<0 .
\end{aligned}
$$

Again in view of Lemmas 2 and 3, the following two matrix inequalities are obvious:

$$
\begin{aligned}
& M_{A}(i) H_{A}(t, i) N_{A}(i) X(i)+X(i) N_{A}^{T}(i) H_{A}^{T}(t, i) M_{A}^{T}(i) \\
& \leq R_{A 1}(i) Q_{A}^{-1}(i) R_{A 1}^{T}(i)+\varepsilon_{A}^{-2}(i) X(i) N_{A}^{T}(i) N_{A}(i) X(i), \\
& M_{B}(i) H_{B}(t, i) N_{B}(i) K(i) X(i) \\
& \quad+X(i) K^{T}(i) N_{B}^{T}(i) H_{B}^{T}(t, i) M_{B}^{T}(i) \\
& \leq R_{B 1}(i) Q_{B}^{-1}(i) R_{B 1}^{T}(i) \\
& \quad+\varepsilon_{B}^{-2}(i) X(i) K^{T}(i) N_{B}^{T}(i) N_{B}(i) K(i) X(i),
\end{aligned}
$$

where

$$
\begin{gathered}
R_{A 1}(i)=\varepsilon_{A}(i) M_{A}(i)+\varepsilon_{A}^{-1}(i) X(i) N_{A}^{T}(i) G_{A}(i), \\
Q_{A}(i)=I-G_{A}^{T}(i) G_{A}(i), \\
R_{B 1}(i)=\varepsilon_{B}(i) M_{B}(i)+\varepsilon_{B}^{-1}(i) X(i) K^{T}(i) N_{B}^{T}(i) G_{B}(i), \\
Q_{B}(i)=I-G_{B}^{T}(i) G_{B}(i) .
\end{gathered}
$$

Setting

$$
\begin{gathered}
\mathbb{X}(i)=\operatorname{diag}\{X(1), \ldots, X(i-1), X(i+1), \ldots, X(N)\}, \\
\mathbb{S}(X(i))=X(i)\left[\sqrt{\lambda_{i 1}} I, \ldots, \sqrt{\lambda_{i, i-1}} I, \sqrt{\lambda_{i, i+1}} I, \ldots, \sqrt{\lambda_{i N}} I\right] .
\end{gathered}
$$

Then $X(i)\left[\sum_{j=1}^{N} \lambda_{i j} X^{-1}(j)\right] X(i)$ can be rewritten as follows:

$$
\begin{aligned}
X(i)\left[\sum_{j=1}^{N} \lambda_{i j} X^{-1}(j)\right] X(i) \\
\quad=\lambda_{i i} X(i)+\mathbb{S}(X(i)) \mathbb{X}^{-1}(i) \mathbb{S}^{T}(X(i)) .
\end{aligned}
$$

Let $Y(i)=K(i) X(i)$, and by means of the matrix inequalities (31) and (34), the closed-loop system (27) will be stochastically stable if the following matrix inequality holds:

$$
\begin{aligned}
X(i) & A^{T}(i)+A(i) X(i)+Y^{T}(i) B^{T}(i)+B(i) Y(i) \\
& +\lambda_{i i} X(i)+R_{A 1}(i) Q_{A}^{-1}(i) R_{A 1}^{T}(i) \\
& +\varepsilon_{A}^{-2}(i) X(i) N_{A}^{T}(i) N_{A}(i) X(i) \\
& +R_{B 1}(i) Q_{B}^{-1}(i) R_{B 1}^{T}(i) \\
& +\varepsilon_{B}^{-2}(i) Y^{T}(i) N_{B}^{T}(i) N_{B}(i) Y(i) \\
& +X(i) W^{T}(i) X^{-1}(i) W(i) X(i) \\
& +\mathbb{S}(X(i)) \mathbb{X}^{-1}(i) \mathbb{S}^{T}(X(i))<0 .
\end{aligned}
$$

After using the Schur complement, we can get the LMIs (25), which end the proof of Theorem 5 .

4.2. Dynamic Output Feedback Stochastic Stabilization. In Section 4.1, the design method of state feedback controller had been given under that the state vector $x(t)$ and the mode $r(t)$ are available at each time $t$. However, some of the state variables are not measurable by their construction or the lack of appropriate sensors to give information. Alternatively we can use dynamic output feedback controller that uses the system's measurement to design and to compute the control gains in this subsection.

In this subsection, we discuss how to design the dynamic output feedback control law for the following Itô stochastic uncertain MJLS:

$$
\begin{gathered}
\begin{array}{c}
d x(t)=A(t, r(t)) x(t) d t+B(t, r(t)) u(t) d t \\
+W(r(t)) x(t) d \omega(t), \\
y(t)=C(t, r(t)) x(t), \quad x(0)=x_{0}, \quad r(0)=r_{0},
\end{array}
\end{gathered}
$$

where $C(t, r(t)), r(t)=i \in \overline{\mathbb{N}}$ are system matrices with compatible dimensions that depend on $r(t)$ and $t$, and

$$
\begin{gathered}
C(t, i)=C(i)+M_{C}(i) H_{C}(t, i) N_{C}(i), \\
H_{C}(t, i)=\Sigma_{C}(t, i)\left[I-G_{C}(i) \Sigma_{C}(t, i)\right]^{-1},
\end{gathered}
$$

where $C(i), M_{C}(i), N_{C}(i)$, and $G_{C}(i)$ are known real constant matrices with appropriate dimensions. $\Sigma_{C}(t, i)$ is an unknown real matrix function with Lebesgue-measurable elements, satisfying $\Sigma_{C}^{T}(t, i) \Sigma_{C}(t, i) \leq I$ with $I$ being the identity matrix. The dynamic output feedback control law is to be described by the following structure:

$$
\begin{gathered}
\dot{x}_{c}(t)=K_{A}(r(t)) x_{c}(t)+K_{B}(r(t)) y(t), \\
u(t)=K_{C}(r(t)) x_{c}(t), \quad x_{c}(0)=0, \quad r(0)=0,
\end{gathered}
$$

where $x_{c}(t) \in \mathbb{R}^{n}$ is the controller state and $K_{A}(r(t))$, $K_{B}(r(t))$, and $K_{C}(r(t))$ are the controller gains to be determined. 
Theorem 6. If there exists a set of matrices $\left\{X^{T}(i)=X(i)>\right.$ $0 \mid i=1,2, \ldots, N\},\{Y(i)>0 \mid i=1,2, \ldots, N\}$, $\left\{\mathbb{k}_{B}(i) \mid i=1,2, \ldots, N\right\}$, and $\left\{\mathbb{k}_{C}(i) \mid i=1,2, \ldots, N\right\}$ and a set of positive scalars $\left\{\varepsilon_{A}(i) \mid i=1,2, \ldots, N\right\},\left\{\varepsilon_{B}(i) \mid\right.$ $i=1,2, \ldots, N\}$, and $\left\{\varepsilon_{C}(i) \mid i=1,2, \ldots, N\right\}$ such that the following sets of inequalities (39)-(41) hold for each $i \in \overline{\mathbb{N}}$ and all admissible linear fractional uncertainties, then the dynamic output feedback controller (38) with control gain (43) can stabilize stochastically the Itô stochastic uncertain MJLS in (36)

$$
\begin{aligned}
& {\left[\begin{array}{cccccccc}
J_{d 1}(i) & * & * & * & * & * & * & * \\
R_{A 2}^{T}(i) & -Q_{A}(i) & 0 & 0 & 0 & 0 & 0 & 0 \\
R_{B 2}^{T}(i) & 0 & -Q_{B}(i) & 0 & 0 & 0 & 0 & 0 \\
\varepsilon_{C}^{-1}(i) G_{C}^{T}(i) N_{C}(i) Y(i) & 0 & 0 & -Q_{C}(i) & 0 & 0 & 0 & 0 \\
\varepsilon_{A}^{-1}(i) N_{A}(i) Y(i) & 0 & 0 & 0 & -I & 0 & 0 & 0 \\
\varepsilon_{B}^{-1}(i) N_{B}(i) \mathbb{k}_{C}(i) & 0 & 0 & 0 & 0 & -I & 0 & 0 \\
\varepsilon_{C}^{-1}(i) N_{C}(i) Y(i) & 0 & 0 & 0 & 0 & 0 & -I & 0 \\
\mathbb{S}^{T}(Y(i)) & 0 & 0 & 0 & 0 & 0 & 0 & -\mathbb{Y}(i)
\end{array}\right]<0,}
\end{aligned}
$$

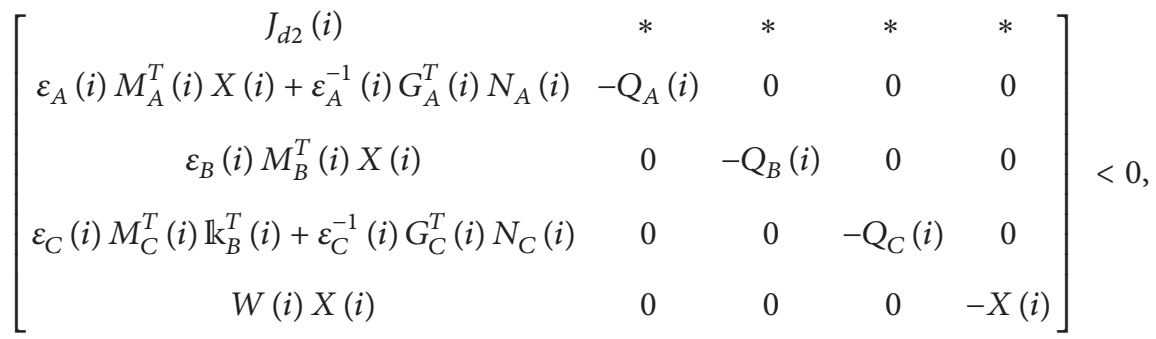

$$
\begin{aligned}
& {\left[\begin{array}{cc}
Y(i) & I \\
I & X(i)
\end{array}\right]>0}
\end{aligned}
$$

where $*$ represents the blocks that are induced by symmetry and

$$
\begin{gathered}
J_{d 1}(i)=A(i) Y(i)+Y^{T}(i) A(i)+B(i) \mathbb{k}_{C}(i) \\
+\mathbb{k}_{C}^{T}(i) B^{T}(i)+\lambda_{i i} Y(i) \\
J_{d 2}(i)=X(i) A(i)+\mathbb{k}_{B}(i) C(i)+A^{T}(i) X(i)+C^{T}(i) \mathbb{k}_{B}^{T}(i) \\
+\sum_{j=1}^{N} \lambda_{i j} X(j)+\varepsilon_{A}^{-2}(i) N_{A}^{T}(i) N_{A}(i) \\
+\varepsilon_{C}^{-2}(i) N_{C}^{T}(i) N_{C}(i)
\end{gathered}
$$

$\mathbb{S}(Y(i))$

$$
\begin{gathered}
\mathbb{Y}(i)=\operatorname{diag}\{Y(1), \ldots, Y(i-1), Y(i+1), \ldots, Y(N)\}, \\
R_{A 2}(i)=\varepsilon_{A}(i) M_{A}(i)+\varepsilon_{A}^{-1}(i) Y^{T}(i) N_{A}^{T}(i) G_{A}(i), \\
R_{B 2}(i)=\varepsilon_{B}(i) M_{B}(i)+\varepsilon_{B}^{-1}(i) \mathbb{k}_{C}^{T}(i) N_{B}^{T}(i) G_{B}(i), \\
Q_{A}(i)=I-G_{A}^{T}(i) G_{A}(i), \\
Q_{B}(i)=I-G_{B}^{T}(i) G_{B}(i), \\
Q_{C}(i)=I-G_{C}^{T}(i) G_{C}(i) .
\end{gathered}
$$

$$
=Y(i)\left[\sqrt{\lambda_{i 1}} I, \ldots, \sqrt{\lambda_{i, i-1}} I, \sqrt{\lambda_{i, i+1}} I, \ldots, \sqrt{\lambda_{i N}} I\right],
$$




$$
K_{A}(i)=\left[X(i)-Y^{-1}(i)\right]^{-1}\left[\begin{array}{c}
A^{T}(i) Y^{-1}(i)+X(i) A(i)+\mathbb{k}_{\mathbb{B}}(i) C(i)+X(i) B(i) \mathbb{k}_{\mathbb{C}}(i) Y^{-1}(i) \\
\quad+\sum_{j=1}^{N} \lambda_{i j} Y^{-1}(j)+N_{A}^{T}(i) G_{A}(i) Q_{A}^{-1}(i) M_{A}^{T}(i) Y^{-1}(i) \\
\\
+X(i) M_{A}(i) Q_{A}^{-1}(i) G_{A}^{T}(i) N_{A}(i) \\
+X(i) M_{B}(i) Q_{B}^{-1}(i) G_{B}^{T}(i) N_{B}(i) \mathbb{k}_{\mathbb{C}}(i) Y^{-1}(i) \\
+\mathbb{k}_{\mathbb{B}}(i) M_{C}(i) Q_{C}^{-1}(i) G_{C}^{T}(i) N_{C}(i) \\
+\varepsilon_{A}^{2}(i) X(i) M_{A}(i) Q_{A}^{-1}(i) M_{A}^{T}(i) Y^{-1}(i) \\
+\varepsilon_{A}^{-2}(i) N_{A}^{T}(i) G_{A}(i) Q_{A}^{-1}(i) G_{A}^{T}(i) N_{A}(i)+\varepsilon_{A}^{-2}(i) N_{A}^{T}(i) N_{A}(i) \\
+\varepsilon_{B}^{2}(i) X(i) M_{B}(i) Q_{B}^{-1}(i) M_{B}^{T}(i) Y^{-1}(i) \\
+\varepsilon_{C}^{-2}(i) N_{C}^{T}(i) G_{C}(i) Q_{C}^{-1}(i) G_{C}^{T}(i) N_{C}(i) \\
+\varepsilon_{C}^{-2}(i) N_{C}^{T}(i) N_{C}(i)+W^{T}(i) X(i) W(i)
\end{array}\right],
$$

Proof. Combining the dynamic system (36) with controller dynamics (38), we can obtain the following extended dynamics:

$$
d \eta(t)=\widehat{A}(t, r(t)) \eta(t) d t+\widehat{W}(r(t)) \eta(t) d \omega(t),
$$

where $\widehat{A}(t, r(t))$ and $\widehat{W}(r(t)), r(t)=i \in \overline{\mathbb{N}}$ are system matrices with compatible dimensions that depend on $r(t)$ and $t$, and

$$
\begin{aligned}
& \eta(t)=\left[x^{T}(t), x_{c}^{T}(t)\right]^{T} \in \mathbb{R}^{2 n}, \\
& \widehat{A}(t, i)=\widehat{A}(i)+\Delta \hat{A}_{A}(t, i)+\Delta \widehat{A}_{B}(t, i)+\Delta \hat{A}_{C}(t, i), \\
& \hat{A}(i)=\left[\begin{array}{cc}
A(i) & B(i) K_{C}(i) \\
K_{B}(i) C(i) & K_{A}(i)
\end{array}\right], \\
& \Delta \hat{A}_{A}(t, i)=\left[\begin{array}{cc}
M_{A}(i) H_{A}(t, i) N_{A}(i) & 0 \\
0 & 0
\end{array}\right], \\
& \widehat{W}(i)=\left[\begin{array}{cc}
W(i) & 0 \\
0 & 0
\end{array}\right] \text {, } \\
& \Delta \widehat{A}_{B}(t, i)=\left[\begin{array}{cc}
0 & M_{B}(i) H_{B}(t, i) N_{B}(i) K_{C}(i) \\
0 & 0
\end{array}\right], \\
& \Delta \hat{A}_{C}(t, i)=\left[\begin{array}{cc}
0 & 0 \\
K_{B}(i) M_{C}(i) H_{C}(t, i)(t) N_{C}(i) & 0
\end{array}\right] .
\end{aligned}
$$

Applying Theorem 4, the dynamics (44) is stochastically stable if there exists a set of symmetric and positive-definite matrices $\{P(i) \mid i=1,2, \ldots, N\}$ such that the following inequalities hold for every $i \in \overline{\mathbb{N}}$ :

$$
\begin{aligned}
& \widehat{A}^{T}(t, i) P(i)+P(i) \widehat{A}(t, i)+\widehat{W}^{T}(i) P(i) \widehat{W}(i) \\
& \quad+\sum_{j=1}^{N} \lambda_{i j} P(j)<0 .
\end{aligned}
$$

Let $Q_{A}(i)=I-G_{A}^{T}(i) G_{A}(i), Q_{B}(i)=I-G_{B}^{T}(i) G_{B}(i)$, and $Q_{C}(i)=I-G_{C}^{T}(i) G_{C}(i)$, and using Lemma 3, we have

$$
\begin{aligned}
& \Delta \widehat{A}_{A}(t, i) \\
& =\left[\begin{array}{cc}
M_{A}(i) & 0 \\
0 & 0
\end{array}\right]\left[\begin{array}{cc}
Q_{A}^{-1}(i) & 0 \\
0 & 0
\end{array}\right]\left[\begin{array}{cc}
G_{A}^{T}(i) & 0 \\
0 & 0
\end{array}\right]\left[\begin{array}{cc}
N_{A}(i) & 0 \\
0 & 0
\end{array}\right] \\
& +\left[\begin{array}{cc}
M_{A}(i) & 0 \\
0 & 0
\end{array}\right]\left[\begin{array}{cc}
Q_{A}^{-1 / 2}(i) & 0 \\
0 & 0
\end{array}\right]\left[\begin{array}{cc}
\Pi_{A}(t, i) & 0 \\
0 & 0
\end{array}\right]\left[\begin{array}{cc}
N_{A}(i) & 0 \\
0 & 0
\end{array}\right] \\
& \stackrel{\text { def }}{=} \widehat{M}_{A}(i) \widehat{Q}_{A}^{-1}(i) \widehat{G}_{A}^{T}(i) \hat{N}_{A}(i) \\
& +\hat{M}_{A}(i) \widehat{Q}_{A}^{-1 / 2}(i) \hat{\Pi}_{A}(t, i) \hat{N}_{A}(i),
\end{aligned}
$$

$\Delta \widehat{A}_{B}(t, i)$

$=\left[\begin{array}{cc}0 & M_{B}(i) \\ 0 & 0\end{array}\right]\left[\begin{array}{cc}0 & 0 \\ 0 & Q_{B}^{-1}(i)\end{array}\right]\left[\begin{array}{cc}0 & 0 \\ 0 & G_{B}^{T}(i)\end{array}\right]\left[\begin{array}{cc}0 & 0 \\ 0 & N_{B}(i) K_{C}(i)\end{array}\right]$

$+\left[\begin{array}{cc}0 & M_{B}(i) \\ 0 & 0\end{array}\right]\left[\begin{array}{cc}0 & 0 \\ 0 & Q_{B}^{-1 / 2}(i)\end{array}\right]\left[\begin{array}{cc}0 & 0 \\ 0 & \Pi_{B}(t, i)\end{array}\right]\left[\begin{array}{cc}0 & 0 \\ 0 & N_{B}(i) K_{C}(i)\end{array}\right]$

$\stackrel{\text { def }}{=} \widehat{M}_{B}(i) \widehat{Q}_{B}^{-1}(i) \widehat{G}_{B}^{T}(i) \widehat{N}_{B}(i)+\widehat{M}_{B}(i) \widehat{Q}_{B}^{-1 / 2}(i) \widehat{\Pi}_{B}(t, i) \widehat{N}_{B}(i)$,

$\Delta \bar{A}_{C}(t, i)$

$=\left[\begin{array}{cc}0 & 0 \\ K_{B}(i) M_{C}(i) & 0\end{array}\right]\left[\begin{array}{cc}Q_{C}^{-1}(i) & 0 \\ 0 & 0\end{array}\right]\left[\begin{array}{cc}G_{C}^{T}(i) & 0 \\ 0 & 0\end{array}\right]\left[\begin{array}{cc}N_{C}(i) & 0 \\ 0 & 0\end{array}\right]$

$$
+\left[\begin{array}{cc}
0 & 0 \\
K_{B}(i) M_{C}(i) & 0
\end{array}\right]\left[\begin{array}{cc}
Q_{C}^{-1 / 2}(i) & 0 \\
0 & 0
\end{array}\right]\left[\begin{array}{cc}
\Pi_{C}(t, i) & 0 \\
0 & 0
\end{array}\right]\left[\begin{array}{cc}
N_{C}(i) & 0 \\
0 & 0
\end{array}\right]
$$

$\stackrel{\text { def }}{=} \widehat{M}_{C}(i) \widehat{Q}_{C}^{-1}(i) \widehat{G}_{C}^{T}(i) \hat{N}_{C}(i)+\hat{M}_{C}(i) \widehat{Q}_{C}^{-1 / 2}(i) \hat{\Pi}_{C}(t, i) \hat{N}_{C}(i)$. 
In view of (47) and Lemma 2, we have

$$
\begin{aligned}
& P(i) \Delta \widehat{A}_{A}(t, i)+\Delta \widehat{A}_{A}^{T}(t, i) P(i) \\
& \leq P(i) \widehat{M}_{A}(i) \widehat{Q}_{A}^{-1}(i) \widehat{G}_{A}^{T}(i) \widehat{N}_{A}(i) \\
& +\widehat{N}_{A}^{T}(i) \hat{G}_{A}(i) \widehat{Q}_{A}^{-1}(i) \hat{M}_{A}^{T}(i) P(i) \\
& +\varepsilon_{A}^{-2}(i) \widehat{N}_{A}^{T}(i) \widehat{N}_{A}(i) \\
& +\varepsilon_{A}^{2}(i) P(i) \widehat{M}_{A}(i) \widehat{Q}_{A}^{-1}(i) \widehat{M}_{A}^{T}(i) P(i) \\
& +\varepsilon_{A}^{-2}(i) \hat{N}_{A}^{T}(i) \widehat{G}_{A}(i) \widehat{Q}_{A}^{-1}(i) \widehat{G}_{A}^{T}(i) \hat{N}_{A}(i), \\
& P(i) \Delta \widehat{A}_{B}(t, i)+\Delta \widehat{A}_{B}^{T}(t, i) P(i) \\
& \leq P(i) \widehat{M}_{B}(i) \widehat{N Q}_{B}^{-1}(i) \widehat{G}_{B}^{T}(i) \widehat{N}_{B}(i) \\
& +\widehat{N}_{B}^{T}(i) \widehat{G}_{B}(i) \widehat{Q}_{B}^{-1}(i) \widehat{M}_{B}^{T}(i) P(i) \\
& +\varepsilon_{B}^{-2}(i) \widehat{N}_{B}^{T}(i) \widehat{N}_{B}(i) \\
& +\varepsilon_{B}^{2}(i) P(i) \widehat{M}_{B}(i) \widehat{Q}_{B}^{-1}(i) \widehat{M}_{B}^{T}(i) P(i) \\
& +\varepsilon_{B}^{-2}(i) \widehat{N}_{B}^{T}(i) \widehat{G}_{B}(i) \widehat{Q}_{B}^{-1}(i) \widehat{G}_{B}^{T}(i) \widehat{N}_{B}(i), \\
& P(i) \Delta \widehat{A}_{C}(t, i)+\Delta \widehat{A}_{C}^{T}(t, i) P(i) \\
& \leq P(i) \widehat{M}_{C}(i) \hat{Q}_{C}^{-1}(i) \widehat{G}_{C}^{T}(i) \widehat{N}_{C}(i) \\
& +\widehat{N}_{C}^{T}(i) \widehat{G}_{C}(i) \widehat{Q}_{C}^{-1}(i) \hat{M}_{C}^{T}(i) P(i) \\
& +\varepsilon_{C}^{-2}(i) \hat{N}_{C}^{T}(i) \widehat{N}_{C}(i) \\
& +\varepsilon_{C}^{2}(i) P(i) \widehat{M}_{C}(i) \widehat{Q}_{C}^{-1}(i) \widehat{M}_{C}^{T}(i) P(i) \\
& +\varepsilon_{C}^{-2}(i) \widehat{N}_{c}^{T}(i) \widehat{G}_{C}(i) \widehat{Q}_{C}^{-1}(i) \widehat{G}_{C}^{T}(i) \widehat{N}_{C}(i) .
\end{aligned}
$$

Based on the matrix inequality (48), the condition (46) will be satisfied if the following holds:

$$
\begin{aligned}
\widehat{A}^{T}(i) & P(i)+P(i) \hat{A}(i)+\sum_{j=1}^{N} \lambda_{i j} P(j)+\widehat{W}^{T}(i) P(i) \widehat{W}(i) \\
& +P(i) \hat{M}_{A}(i) \widehat{Q}_{A}^{-1}(i) \widehat{G}_{A}^{T}(i) \widehat{N}_{A}(i) \\
& +\widehat{N}_{A}^{T}(i) \widehat{G}_{A}(i) \widehat{Q}_{A}^{-1}(i) \widehat{M}_{A}^{T}(i) P(i)
\end{aligned}
$$

$$
\begin{aligned}
& +\varepsilon_{A}^{-2}(i) \hat{N}_{A}^{T}(i) \hat{N}_{A}(i)+\varepsilon_{B}^{-2}(i) \hat{N}_{B}^{T}(i) \hat{N}_{B}(i) \\
& +\varepsilon_{A}^{2}(i) P(i) \widehat{M}_{A}(i) \widehat{Q}_{A}^{-1}(i) \widehat{M}_{A}^{T}(i) P(i) \\
& +\varepsilon_{A}^{-2}(i) \widehat{N}_{A}^{T}(i) \widehat{G}_{A}(i) \widehat{Q}_{A}^{-1}(i) \widehat{G}_{A}^{T}(i) \hat{N}_{A}(i) \\
& +P(i) \widehat{M}_{B}(i) \widehat{Q}_{B}^{-1}(i) \widehat{G}_{B}^{T}(i) \widehat{N}_{B}(i) \\
& +\widehat{N}_{B}^{T}(i) \widehat{G}_{B}(i) \hat{Q}_{B}^{-1}(i) \widehat{M}_{B}^{T}(i) P(i) \\
& +\varepsilon_{B}^{2}(i) P(i) \widehat{M}_{B}(i) \widehat{Q}_{B}^{-1}(i) \widehat{M}_{B}^{T}(i) P(i) \\
& +\varepsilon_{B}^{-2}(i) \widehat{N}_{B}^{T}(i) \widehat{G}_{B}(i) \widehat{Q}_{B}^{-1}(i) \widehat{G}_{B}^{T}(i) \widehat{N}_{B}(i) \\
& +P(i) \widehat{M}_{C}(i) \widehat{Q}_{C}^{-1}(i) \widehat{G}_{C}^{T}(i) \widehat{N}_{C}(i) \\
& +\hat{N}_{C}^{T}(i) \widehat{G}_{C}(i) \widehat{Q}_{C}^{-1}(i) \widehat{M}_{C}^{T}(i) P(i) \\
& +\varepsilon_{C}^{-2}(i) \widehat{N}_{C}^{T}(i) \widehat{N}_{C}(i) \\
& +\varepsilon_{C}^{2}(i) P(i) \hat{M}_{C}(i) \widehat{Q}_{C}^{-1}(i) \widehat{M}_{C}^{T}(i) P(i) \\
& +\varepsilon_{C}^{-2}(i) \widehat{N}_{c}^{T}(i) \widehat{G}_{C}(i) \widehat{Q}_{C}^{-1}(i) \widehat{G}_{C}^{T}(i) \widehat{N}_{C}(i)<0
\end{aligned}
$$

It is obvious that inequality (49) is nonlinear in the design parameters $K_{A}(i), K_{B}(i), K_{C}(i)$, and $P(i), i \in \overline{\mathbb{N}}$. In order to transform inequality (49) into an LMI, define $P(i), L(i), U(i)$, and $V(i)$ as follows:

$$
\begin{gathered}
P(i)=\left[\begin{array}{ll}
P_{1}(i) & P_{2}(i) \\
P_{2}^{T}(i) & P_{3}(i)
\end{array}\right], \\
L(i)=\left(P_{1}(i)-P_{2}(i) P_{3}^{-1}(i) P_{2}^{T}(i)\right)^{-1}, \\
U(i)=\left[\begin{array}{ll}
L(i) & I \\
L(i) & 0
\end{array}\right], \\
V(i)=\left[\begin{array}{cc}
I & 0 \\
0 & -P_{3}^{-1}(i) P_{2}^{T}(i)
\end{array}\right],
\end{gathered}
$$

where $P_{1}(i), P_{3}(i)$ are symmetric and positive-define matrices.

Before and after multiply inequality (49) by $U^{T}(i) V^{T}(i)$ and $V(i) U(i)$, respectively, we have 


$$
\begin{aligned}
& U^{T}(i) V^{T}(i) P(i) \widehat{A}(i) V(i) U(i) \\
& =\left[\begin{array}{cc}
A(i) L(i)-B(i) K_{C}(i) P_{3}^{-1}(i) P_{2}^{T}(i) L(i) & A(i) \\
\left(\begin{array}{c}
P_{1}(i) A(i) L(i)-P_{1}(i) B(i) K_{C}(i) P_{3}^{-1}(i) P_{2}^{T}(i) L(i) \\
-P_{2}(i) K_{A}(i) P_{3}^{-1}(i) P_{2}^{T}(i) L(i)+P_{2}(i) K_{B}(i) C(i) L(i)
\end{array}\right) & P_{1}(i) A(i)+P_{2}(i) K_{B}(i) C(i)
\end{array}\right], \\
& U^{T}(i) V^{T}(i) P(j) V(i) U(i) \\
& =\left[\begin{array}{cc}
L^{T}(i) L^{-1}(j) L(i)+L^{T}(i)\left(P_{2}(i) P_{3}^{-1}(i) P_{3}(j)-P_{2}(j)\right) \\
\times P_{3}^{-1}(j)\left(P_{2}(i) P_{3}^{-1}(i) P_{3}(j)-P_{2}(j)\right)^{T} L(i) \\
\left(P_{1}(j)-P_{2}(j) P_{3}^{-1}(i) P_{2}^{T}(i)\right) L(i) & L^{T}(i)\left(P_{1}(j)-P_{2}(j) P_{3}^{-1}(i) P_{2}^{T}(i)\right)^{T} \\
P_{1}(j)
\end{array}\right],
\end{aligned}
$$$$
U^{T}(i) V^{T}(i) P(i) \widehat{M}_{A}(i) \widehat{Q}_{A}^{-1}(i) \widehat{G}_{A}^{T}(i) \widehat{N}_{A}(i) V(i) U(i)
$$$$
=\left[\begin{array}{cc}
M_{A}(i) Q_{A}^{-1}(i) G_{A}^{T}(i) N_{A}(i) L(i) & M_{A}(i) Q_{A}^{-1}(i) G_{A}^{T}(i) N_{A}(i) \\
P_{1}(i) M_{A}(i) Q_{A}^{-1}(i) G_{A}^{T}(i) N_{A}(i) L(i) & P_{1}(i) M_{A}(i) Q_{A}^{-1}(i) G_{A}^{T}(i) N_{A}(i)
\end{array}\right],
$$$$
U^{T}(i) V^{T}(i) P(i) \widehat{M}_{A}(i) \widehat{Q}_{A}^{-1}(i) \widehat{M}_{A}^{T}(i) P(i) V(i) U(i)
$$$$
=\left[\begin{array}{cc}
M_{A}(i) Q_{A}^{-1}(i) M_{A}^{T}(i) & M_{A}(i) Q_{A}^{-1}(i) M_{A}^{T}(i) P_{1}(i) \\
P_{1}(i) M_{A}(i) Q_{A}^{-1}(i) M_{A}^{T}(i) & P_{1}(i) M_{A}(i) Q_{A}^{-1}(i) M_{A}^{T}(i) P_{1}(i)
\end{array}\right],
$$$$
U^{T}(i) V^{T}(i) \widehat{N}_{A}^{T}(i) \widehat{G}_{A}(i) \widehat{Q}_{A}^{-1}(i) \widehat{G}_{A}^{T}(i) \hat{N}_{A}(i) V(i) U(i)
$$$$
=\left[\begin{array}{cc}
L^{T}(i) N_{A}^{T}(i) G_{A}(i) Q_{A}^{-1}(i) G_{A}^{T}(i) N_{A}(i) L(i) & L^{T}(i) N_{A}^{T}(i) G_{A}(i) Q_{A}^{-1}(i) G_{A}^{T}(i) N_{A}(i) \\
N_{A}^{T}(i) G_{A}(i) Q_{A}^{-1}(i) G_{A}^{T}(i) N_{A}(i) L(i) & N_{A}^{T}(i) G_{A}(i) Q_{A}^{-1}(i) G_{A}^{T}(i) N_{A}(i)
\end{array}\right],
$$$$
U^{T}(i) V^{T}(i) \hat{N}_{A}^{T}(i) \hat{N}_{A}(i) V(i) U(i)=\left[\begin{array}{cc}
L^{T}(i) N_{A}^{T}(i) N_{A}(i) L(i) & L^{T}(i) N_{A}^{T}(i) N_{A}(i) \\
N_{A}^{T}(i) N_{A}(i) L(i) & N_{A}^{T}(i) N_{A}(i)
\end{array}\right],
$$$$
U^{T}(i) V^{T}(i) P(i) \widehat{M}_{B}(i) \widehat{Q}_{B}^{-1}(i) \widehat{G}_{B}^{T}(i) \widehat{N}_{B}^{T}(i) V(i) U(i)
$$

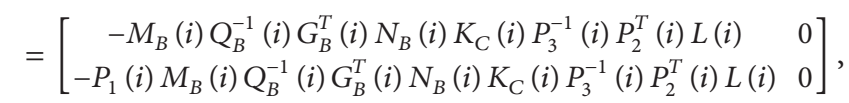$$
U^{T}(i) V^{T}(i) P(i) \widehat{M}_{B}(i) \widehat{Q}_{B}^{-1}(i) \widehat{M}_{B}^{T}(i) P(i) V(i) U(i)
$$$$
=\left[\begin{array}{cc}
M_{B}(i) Q_{B}^{-1}(i) M_{B}^{T}(i) & M_{B}(i) Q_{B}^{-1}(i) M_{B}^{T}(i) P_{1}(i) \\
P_{1}(i) M_{B}(i) Q_{B}^{-1}(i) M_{B}^{T}(i) & P_{1}(i) M_{B}(i) Q_{B}^{-1}(i) M_{B}^{T}(i) P_{1}(i)
\end{array}\right],
$$

$$
U^{T}(i) V^{T}(i) \widehat{N}_{B}^{T}(i) \widehat{G}_{B}(i) \widehat{Q}_{B}^{-1}(i) \widehat{G}_{B}^{T}(i) \widehat{N}_{B}(i) V(i) U(i)
$$$$
=\left[\begin{array}{cc}
L^{T}(i) P_{2}(i) P_{3}^{-1}(i) K_{C}^{T}(i) N_{B}^{T}(i) G_{B}(i) Q_{B}^{-1}(i) G_{B}^{T}(i) N_{B}(i) K_{C}(i) P_{3}^{-1}(i) P_{2}^{T}(i) L(i) & 0 \\
0
\end{array}\right] \text {, }
$$$$
U^{T}(i) V^{T}(i) \widehat{N}_{B}^{T}(i) \hat{N}_{B}(i) V(i) U(i)
$$$$
=\left[\begin{array}{cc}
L^{T}(i) P_{2}(i) P_{3}^{-1}(i) K_{C}^{T}(i) N_{B}^{T}(i) N_{B}(i) K_{C}(i) P_{3}^{-1}(i) P_{2}^{T}(i) L(i) & 0 \\
0 & 0
\end{array}\right],
$$

$U^{T}(i) V^{T}(i) P(i) \widehat{M}_{C}(i) \widehat{Q}_{C}^{-1}(i) \widehat{G}_{C}^{T}(i) \widehat{N}_{C}^{T}(i) V(i) U(i)$

$$
=\left[\begin{array}{c}
0 \\
P_{2}(i) K_{B}(i) M_{C}(i) Q_{C}^{-1}(i) G_{C}^{T}(i) N_{C}(i) L(i) \\
P_{2}(i) K_{B}(i) M_{C}(i) Q_{C}^{-1}(i) G_{C}^{T}(i) N_{C}(i)
\end{array}\right],
$$

$U^{T}(i) V^{T}(i) P(i) \hat{M}_{C}(i) \hat{Q}_{C}^{-1}(i) \hat{M}_{C}^{T}(i) P(i) V(i) U(i)=\left[\begin{array}{cc}0 & 0 \\ 0 & P_{2}(i) K_{B}(i) M_{C}(i) Q_{C}^{-1}(i) M_{C}^{T}(i) K_{B}^{T}(i) P_{2}^{T}(i)\end{array}\right]$, 


$$
\begin{gathered}
U^{T}(i) V^{T}(i) \hat{N}_{C}^{T}(i) \widehat{G}_{C}(i) \widehat{Q}_{C}^{-1}(i) \widehat{G}_{C}^{T}(i) \hat{N}_{C}(i) V(i) U(i) \\
=\left[\begin{array}{cc}
L^{T}(i) N_{C}^{T}(i) G_{C}(i) Q_{C}^{-1}(i) G_{C}^{T}(i) N_{C}(i) L(i) & L^{T}(i) N_{C}^{T}(i) G_{C}(i) Q_{C}^{-1}(i) G_{C}^{T}(i) N_{C}(i) \\
N_{C}^{T}(i) G_{C}(i) Q_{C}^{-1}(i) G_{C}^{T}(i) N_{C}(i) L(i) & N_{C}^{T}(i) G_{C}(i) Q_{C}^{-1}(i) G_{C}^{T}(i) N_{C}(i)
\end{array}\right], \\
U^{T}(i) V^{T}(i) \hat{N}_{C}^{T}(i) \hat{N}_{C}(i) V(i) U(i)=\left[\begin{array}{cc}
L^{T}(i) N_{C}^{T}(i) N_{C}(i) L(i) & L^{T}(i) N_{C}^{T}(i) N_{C}(i) \\
N_{C}^{T}(i) N_{C}(i) L(i) & N_{C}^{T}(i) N_{C}(i)
\end{array}\right], \\
U^{T}(i) V^{T}(i) \hat{W}^{T}(i) P(i) \hat{W}(i) V(i) U(i)=\left[\begin{array}{cc}
L^{T}(i) W^{T}(i) P_{1}(i) W(i) L(i) & L^{T}(i) W^{T}(i) P_{1}(i) W(i) \\
W^{T}(i) P_{1}(i) W(i) L(i) & W^{T}(i) P_{1}(i) W(i)
\end{array}\right] .
\end{gathered}
$$

Using all these transformation, the condition (49) becomes

$$
\left[\begin{array}{ll}
\widehat{\Xi}_{1}(i) & \Xi_{2}(i) \\
\Xi_{2}^{T}(i) & \Xi_{3}(i)
\end{array}\right]<0,
$$

with

$$
\begin{aligned}
& \widehat{\Xi}_{1}(i) \\
& =\Xi_{1}(i)+\sum_{j=1}^{N} \lambda_{i j} L^{T}(i) \\
& \times\left[P_{2}(i) P_{3}^{-1}(i) P_{3}(j)-P_{2}(j)\right] P_{3}^{-1}(j) \\
& \times\left[P_{2}(i) P_{3}^{-1}(i) P_{3}(j)-P_{2}(j)\right]^{T} L(i) \\
& +L^{T}(i) W^{T}(i) P_{1}(i) W(i) L(i), \\
& \Xi_{1}(i)=A(i) L(i)+L^{T}(i) A^{T}(i) \\
& \text { - B (i) } K_{C}(i) P_{3}^{-1}(i) P_{2}^{T}(i) L(i) \\
& \text { - } L^{T}(i) P_{2}(i) P_{3}^{-1}(i) K_{C}^{T}(i) B^{T}(i) \\
& +\sum_{j=1}^{N} \lambda_{i j} L^{T}(i) L^{-1}(j) L(i) \\
& +M_{A}(i) Q_{A}^{-1}(i) G_{A}^{T}(i) N_{A}(i) L(i) \\
& +L^{T}(i) N_{A}^{T}(i) G_{A}(i) Q_{A}^{-1}(i) M_{A}^{T}(i) \\
& \text { - } M_{B}(i) Q_{B}^{-1}(i) G_{B}^{T}(i) N_{B}(i) \\
& \times K_{C}(i) P_{3}^{-1}(i) P_{2}^{T}(i) L(i) \\
& \text { - } L^{T}(i) P_{2}(i) P_{3}^{-1}(i) K_{C}^{T}(i) N_{B}^{T}(i) \\
& \times G_{B}(i) Q_{B}^{-1}(i) M_{B}^{T}(i) \\
& +\varepsilon_{A}^{2}(i) M_{A}(i) Q_{A}^{-1}(i) M_{A}^{T}(i) \\
& +\varepsilon_{A}^{-2}(i) L^{T}(i) N_{A}^{T}(i) N_{A}(i) L(i)
\end{aligned}
$$




$$
\begin{aligned}
&+L^{T}(i) N_{C}^{T}(i) G_{C}(i) Q_{C}^{-1}(i) M_{C}^{T}(i) K_{B}^{T}(i) P_{2}^{T}(i) \\
&+\varepsilon_{A}^{-2}(i) L^{T}(i) N_{A}^{T}(i) G_{A}(i) Q_{A}^{-1}(i) G_{A}^{T}(i) N_{A}(i) \\
&+\varepsilon_{A}^{-2}(i) L^{T}(i) N_{A}^{T}(i) N_{A}(i) \\
&+\varepsilon_{B}^{2}(i) M_{B}(i) Q_{B}^{-1}(i) M_{B}^{T}(i) P_{1}(i) \\
&+\varepsilon_{C}^{-2}(i) L^{T}(i) N_{C}^{T}(i) G_{C}(i) Q_{C}^{-1}(i) G_{C}^{T}(i) N_{C}(i) \\
&+\varepsilon_{C}^{-2}(i) L^{T}(i) N_{C}^{T}(i) N_{C}(i) \\
& \Xi_{3}(i)= P_{1}(i) A(i)+A^{T}(i) P_{1}(i) \\
&+P_{2}(i) K_{B}(i) C(i)+C^{T}(i) K_{B}^{T}(i) P_{2}^{T}(i) \\
&+\sum_{j=1}^{N} \lambda_{i j} P_{1}(j)+P_{1}(i) M_{A}(i) Q_{A}^{-1}(i) G_{A}^{T}(i) N_{A}(i) \\
&+N_{A}^{T}(i) G_{A}(i) Q_{A}^{-1}(i) M_{A}^{T}(i) P_{1}(i) \\
&+P_{2}(i) K_{B}(i) M_{C}(i) Q_{C}^{-1}(i) G_{C}^{T}(i) N_{C}(i) \\
&+N_{C}^{T}(i) G_{C}(i) Q_{C}^{-1}(i) M_{C}^{T}(i) K_{B}^{T}(i) P_{2}^{T}(i) \\
&+\varepsilon_{A}^{2}(i) P_{1}(i) M_{A}(i) Q_{A}^{-1}(i) M_{A}^{T}(i) P_{1}(i) \\
&+\varepsilon_{A}^{-2}(i) N_{A}^{T}(i) G_{A}(i) Q_{A}^{-1}(i) G_{A}^{T}(i) N_{A}(i) \\
&+\varepsilon_{A}^{-2}(i) N_{A}^{T}(i) N_{A}(i) \\
&+\varepsilon_{B}^{2}(i) P_{1}(i) M_{B}(i) Q_{B}^{-1}(i) M_{B}^{T}(i) P_{1}(i) \\
&+\varepsilon_{C}^{2}(i) P_{2}(i) K_{B}(i) M_{c}(i) Q_{C}^{-1}(i) M_{C}^{T}(i) K_{B}^{T}(i) P_{2}^{T}(i) \\
&+W^{T}(i) P_{1}(i) W(i) \\
&+\varepsilon_{C}^{-2}(i) N_{C}^{T}(i) G_{C}(i) Q_{C}^{-1}(i) G_{C}^{T}(i) N_{C}(i) \\
&+\varepsilon_{C}^{-2}(i) N_{C}^{T}(i) N_{C}(i) \\
&
\end{aligned}
$$

Since $\quad \sum_{j=1}^{N} \lambda_{i j} L^{T}(i)\left[P_{2}(i) P_{3}^{-1}(i) P_{3}(j)\right.$ $\left.P_{2}(j)\right] P_{3}^{-1}(j)\left[P_{2}(i) P_{3}^{-1}(i) P_{3}(j)-P_{2}(j)\right]^{T} L(i) \quad \geq 0$ and $L^{T}(i) W^{T}(i) P_{1}(i) W(i) L(i) \geq 0$, we can obtain the following equivalent condition of (52) as follows:

$$
\left[\begin{array}{ll}
\Xi_{1}(i) & \Xi_{2}(i) \\
\Xi_{2}^{T}(i) & \Xi_{3}(i)
\end{array}\right]<0 .
$$

Let $P_{1}(i)=X(i), P_{2}(i)=Y^{-1}(i)-X(i)$, and $P_{3}(i)=X(i)-$ $Y^{-1}(i)$, then,

$$
\begin{gathered}
L(i)=\left(P_{1}(i)-P_{2}(i) P_{3}^{-1}(i) P_{2}^{T}(i)\right)^{-1}=Y(i), \\
P_{3}^{-1}(i) P_{2}^{T}(i)=-I .
\end{gathered}
$$

Define $\mathbb{k}_{B}(i)$ and $\mathbb{k}_{C}(i)$ by

$$
\begin{gathered}
\mathbb{k}_{B}(i)=P_{2}(i) K_{B}(i)=\left(Y^{-1}(i)-X(i)\right) K_{B}(i), \\
\mathbb{k}_{C}(i)=-K_{C}(i) P_{3}^{-1}(i) P_{2}^{T}(i) L(i)=K_{C}(i) Y(i),
\end{gathered}
$$

then applying all the previous algebraic manipulations, we have

$$
\Xi_{1}(i)=A(i) Y(i)+Y^{T}(i) A^{T}(i)+B(i) \mathbb{k}_{\mathbb{C}}(i)
$$$$
+\mathbb{k}_{C}^{T}(i) B^{T}(i)+\sum_{j=1}^{N} \lambda_{i j} Y^{T}(i) Y^{-1}(j) Y(i)
$$$$
+M_{A}(i) Q_{A}^{-1}(i) G_{A}^{T}(i) N_{A}(i) Y(i)
$$$$
+Y^{T}(i) N_{A}^{T}(i) G_{A}(i) Q_{A}^{-1}(i) M_{A}^{T}(i)
$$$$
+M_{B}(i) Q_{B}^{-1}(i) G_{B}^{T}(i) N_{B}(i) \mathbb{k}_{C}(i)
$$$$
+\mathbb{k}_{C}^{T}(i) N_{B}^{T}(i) G_{B}(i) Q_{B}^{-1}(i) M_{B}^{T}(i)
$$$$
+\varepsilon_{A}^{2}(i) M_{A}(i) Q_{A}^{-1}(i) M_{A}^{T}(i)
$$$$
+\varepsilon_{A}^{-2}(i) Y^{T}(i) N_{A}^{T}(i) G_{A}(i) Q_{A}^{-1}(i) G_{A}^{T}(i) N_{A}(i) Y(i)
$$$$
+\varepsilon_{A}^{-2}(i) Y^{T}(i) N_{A}^{T}(i) N_{A}(i) Y(i)
$$$$
+\varepsilon_{B}^{-2}(i) \mathbb{k}_{C}^{T}(i) N_{B}^{T}(i) G_{B}(i) Q_{B}^{-1}(i) G_{B}^{T}(i) N_{B}(i) \mathbb{k}_{C}(i)
$$$$
+\varepsilon_{B}^{2}(i) M_{B}(i) Q_{B}^{-1}(i) M_{B}^{T}(i)
$$$$
+\varepsilon_{B}^{-2}(i) \mathbb{k}_{C}^{T}(i) N_{B}^{T}(i) N_{B}(i) \mathbb{k}_{C}(i)
$$$$
+\varepsilon_{C}^{-2}(i) Y^{T}(i) N_{C}^{T}(i) G_{C}(i) Q_{C}^{-1}(i) G_{C}^{T}(i) N_{C}(i) Y(i)
$$$$
+\varepsilon_{C}^{-2}(i) Y^{T}(i) N_{C}^{T}(i) N_{C}(i) Y(i),
$$

$$
\Xi_{2}(i)=A(i)+Y^{T}(i) A^{T}(i) X(i)
$$

$+Y^{T}(i) C^{T}(i) \mathbb{k}_{B}^{T}(i)+\mathbb{k}_{C}^{T}(i) B^{T}(i) X(i)$

$+Y^{T}(i) K_{A}^{T}(i)\left(Y^{-1}(i)-X(i)\right)^{T}$

$+\sum_{j=1}^{N} \lambda_{i j} Y^{T}(i) Y^{-1}(j)+Y^{T}(i) W^{T}(i) X(i) W(i)$

$+M_{A}(i) Q_{A}^{-1}(i) G_{A}^{T}(i) N_{A}(i)$

$+Y^{T}(i) N_{A}^{T}(i) G_{A}(i) Q_{A}^{-1}(i) M_{A}^{T}(i) X(i)$

$+\mathbb{k}_{C}^{T}(i) N_{B}^{T}(i) G_{B}(i) Q_{B}^{-1}(i) M_{B}^{T}(i) X(i)$

$+Y^{T}(i) N_{C}^{T}(i) G_{C}(i) Q_{C}^{-1}(i) M_{C}^{T}(i) \mathbb{k}_{B}^{T}(i)$

$+\varepsilon_{A}^{2}(i) M_{A}(i) Q_{A}^{-1}(i) M_{A}^{T}(i) X(i)$

$+\varepsilon_{A}^{-2}(i) Y^{T}(i) N_{A}^{T}(i) G_{A}(i) Q_{A}^{-1}(i) G_{A}^{T}(i) N_{A}(i)$

$+\varepsilon_{A}^{-2}(i) Y^{T}(i) N_{A}^{T}(i) N_{A}(i)$

$+\varepsilon_{B}^{2}(i) M_{B}(i) Q_{B}^{-1}(i) M_{B}^{T}(i) X(i)$

$+\varepsilon_{C}^{-2}(i) Y^{T}(i) N_{C}^{T}(i) G_{C}(i) Q_{C}^{-1}(i) G_{C}^{T}(i) N_{C}(i)$

$+\varepsilon_{C}^{-2}(i) Y^{T}(i) N_{C}^{T}(i) N_{C}(i)$, 


$$
\begin{aligned}
\Xi_{3}(i)= & X(i) A(i)+A^{T}(i) X(i)+\mathbb{k}_{B}(i) C(i) \\
& +C^{T}(i) \mathbb{k}_{B}^{T}(i)+W^{T}(i) X(i) W(i) \\
& +\sum_{j=1}^{N} \lambda_{i j} X(j)+X(i) M_{A}(i) Q_{A}^{-1}(i) G_{A}^{T}(i) N_{A}(i) \\
& +\mathbb{k}_{B}(i) M_{C}(i) Q_{C}^{-1}(i) G_{C}^{T}(i) N_{C}(i) \\
& +N_{C}^{T}(i) G_{C}(i) Q_{C}^{-1}(i) M_{C}^{T}(i) \mathbb{k}_{B}^{T}(i) \\
& +\varepsilon_{A}^{2}(i) X(i) M_{A}(i) Q_{A}^{-1}(i) M_{A}^{T}(i) X(i) \\
& +\varepsilon_{A}^{-2}(i) N_{A}^{T}(i) G_{A}(i) Q_{A}^{-1}(i) G_{A}^{T}(i) N_{A}(i) \\
& +\varepsilon_{A}^{-2}(i) N_{A}^{T}(i) N_{A}(i) \\
& +\varepsilon_{B}^{2}(i) X(i) M_{B}(i) Q_{B}^{-1}(i) M_{B}^{T}(i) X(i) \\
& +\varepsilon_{C}^{2}(i) \mathbb{k}_{B}(i) M_{c}(i) Q_{C}^{-1}(i) M_{C}^{T}(i) \mathbb{k}_{B}^{T}(i) \\
& +N_{A}^{T}(i) G_{A}(i) Q_{A}^{-1}(i) M_{A}^{T}(i) X(i) \\
& +\varepsilon_{C}^{-2}(i) N_{C}^{T}(i) G_{C}(i) Q_{C}^{-1}(i) G_{C}^{T}(i) N_{C}(i) \\
& +\varepsilon_{C}^{-2}(i) N_{C}^{T}(i) N_{C}(i)
\end{aligned}
$$

Using the expression of the controller given by (43), we have $\Xi_{2}(i)=0$. This implies that the stability condition (54) is equivalent to the following conditions:

$$
\begin{aligned}
& \Xi_{1}(i)<0, \\
& \Xi_{3}(i)<0 .
\end{aligned}
$$

Let

$$
\begin{gathered}
\mathbb{S}(Y(i))=Y(i)\left[\sqrt{\lambda_{i 1}} I, \ldots, \sqrt{\lambda_{i, i-1}} I, \sqrt{\lambda_{i, i+1}} I, \ldots, \sqrt{\lambda_{i N}} I\right], \\
\mathbb{Y}(i)=\operatorname{diag}\{Y(1), \ldots, Y(i-1), Y(i+1), \cdots, Y(N)\} .
\end{gathered}
$$

Then,

$$
\begin{aligned}
& \sum_{j=1}^{N} \lambda_{i j} Y^{T}(i) X^{-1}(j) Y(i) \\
& \quad=\lambda_{i i} Y(i)+\mathbb{S}(Y(i)) \mathbb{Y}^{-1}(i) \mathbb{S}^{T}(Y(i)) .
\end{aligned}
$$

According to the Schur complement and

$$
\begin{aligned}
& R_{A 2}(i)=\varepsilon_{A}(i) M_{A}(i)+\varepsilon_{A}^{-1}(i) Y^{T}(i) N_{A}^{T}(i) G_{A}(i), \\
& R_{B 2}(i)=\varepsilon_{B}(i) M_{B}(i)+\varepsilon_{B}^{-1}(i) \mathbb{k}_{C}^{T}(i) N_{B}^{T}(i) G_{B}(i),
\end{aligned}
$$

the stability conditions (58) become (39) and (40). Finally, notice that

$$
U^{T}(i) V^{T}(i) P(i) V(i) U(i)=\left[\begin{array}{cc}
Y(i) & I \\
I & X(i)
\end{array}\right]>0 .
$$

We get (41), which ends the proof of Theorem 6 .

\section{Numerical Example}

The aim of the following two examples is used to illustrate the theoretical results in Section 4.

Example 7 (state feedback stochastic stabilization). Consider the Itô stochastic uncertain MJLS in (22) with $N=2, r(t)$ : $[0, \infty) \rightarrow\{1,2\}$ and

mode no. 1

$$
\begin{gathered}
A(1)=\left[\begin{array}{cc}
1.0 & -0.5 \\
0.1 & 1.0
\end{array}\right], \quad M_{A}(1)=\left[\begin{array}{l}
0.1 \\
0.2
\end{array}\right], \\
N_{A}(1)=\left[\begin{array}{ll}
0.2 & 0.1
\end{array}\right], \quad G_{A}(1)=[0.2] \\
W(1)=\left[\begin{array}{ll}
0.1 & 0.0 \\
0.0 & 0.1
\end{array}\right], \\
B(1)=\left[\begin{array}{ll}
1.0 & 0.0 \\
0.0 & 1.0
\end{array}\right], \quad M_{B}(1)=\left[\begin{array}{l}
0.1 \\
0.2
\end{array}\right], \\
N_{B}(1)=\left[\begin{array}{ll}
0.2 & 0.1
\end{array}\right], \quad G_{B}(1)=[0.2]
\end{gathered}
$$

Mode no. 2

$$
\begin{gathered}
A(2)=\left[\begin{array}{cc}
-0.2 & 0.5 \\
0.0 & -0.25
\end{array}\right], \quad M_{A}(2)=\left[\begin{array}{c}
0.13 \\
0.1
\end{array}\right], \\
N_{A}(2)=\left[\begin{array}{ll}
0.1 & 0.2
\end{array}\right], \quad G_{A}(2)=[0.2] \\
W(2)=\left[\begin{array}{ll}
0.2 & 0.0 \\
0.0 & 0.2
\end{array}\right], \\
B(2)=\left[\begin{array}{ll}
1.0 & 0.0 \\
0.0 & 1.0
\end{array}\right], \quad M_{B}(2)=\left[\begin{array}{c}
0.13 \\
0.1
\end{array}\right] \\
N_{B}(2)=\left[\begin{array}{ll}
0.1 & 0.2
\end{array}\right], \quad G_{B}(2)=[0.2] .
\end{gathered}
$$

The jumping between the different modes is described by the transition rate matrix:

$$
\Lambda=\left[\begin{array}{cc}
-2.0 & 2.0 \\
3.0 & -3.0
\end{array}\right] .
$$

Setting $\varepsilon_{A}(1)=\varepsilon_{A}(2)=0.5$ and $\varepsilon_{B}(1)=\varepsilon_{B}(2)=0.6$. By means of inequality (25), we have

$$
\begin{aligned}
& X(1)=\left[\begin{array}{cc}
0.3445 & -0.0142 \\
-0.0142 & 0.3521
\end{array}\right], \\
& X(2)=\left[\begin{array}{cc}
0.3061 & -0.0107 \\
-0.0107 & 0.3069
\end{array}\right], \\
& Y(1)=\left[\begin{array}{cc}
-0.7333 & 0.0819 \\
0.0819 & -0.7503
\end{array}\right], \\
& Y(2)=\left[\begin{array}{ll}
-0.2168 & -0.0850 \\
-0.0850 & -0.1993
\end{array}\right] .
\end{aligned}
$$

Then applying Theorem 5 , the state feedback controller gains are obtained as follows:

$$
\begin{aligned}
& K(1)=\left[\begin{array}{cc}
-2.1227 & 0.1469 \\
0.1500 & -2.1252
\end{array}\right], \\
& K(2)=\left[\begin{array}{ll}
-0.7197 & -0.3022 \\
-0.3009 & -0.6600
\end{array}\right] .
\end{aligned}
$$


Therefore, the designed state feedback controller (24) is feasible and can guarantee the stochastic stability of the Itô stochastic uncertain MJLS in (22).

Example 8 (dynamic output feedback stochastic stabilization). Consider the Itô stochastic uncertain MJLS in (36) with $N=2, r(t):[0, \infty) \rightarrow\{1,2\}$ and

mode no. 1

$$
\begin{gathered}
A(1)=\left[\begin{array}{cc}
0.2 & -0.6 \\
0.3 & 1.2
\end{array}\right], \quad M_{A}(1)=\left[\begin{array}{l}
0.12 \\
0.23
\end{array}\right], \\
N_{A}(1)=\left[\begin{array}{ll}
0.2 & 0.1
\end{array}\right], \quad G_{A}(1)=[0.5] \\
W(1)=\left[\begin{array}{ll}
0.1 & 0.0 \\
0.0 & 0.1
\end{array}\right], \\
B(1)=\left[\begin{array}{ll}
1.0 & 0.0 \\
0.0 & 1.0
\end{array}\right], \quad M_{B}(1)=\left[\begin{array}{l}
0.12 \\
0.23
\end{array}\right], \\
N_{B}(1)=\left[\begin{array}{ll}
0.2 & 0.1
\end{array}\right], \quad G_{B}(1)=[0.5], \\
C(1)=\left[\begin{array}{ll}
1.0 & 0.0 \\
0.0 & 1.0
\end{array}\right], \quad M_{C}(1)=\left[\begin{array}{l}
0.12 \\
0.23
\end{array}\right], \\
N_{C}(1)=\left[\begin{array}{ll}
0.2 & 0.1
\end{array}\right], \quad G_{C}(1)=[0.5] .
\end{gathered}
$$

Mode no. 2

$$
\begin{gathered}
A(2)=\left[\begin{array}{cc}
-0.3 & 0.4 \\
0.2 & -0.15
\end{array}\right], \quad M_{A}(2)=\left[\begin{array}{l}
0.12 \\
0.15
\end{array}\right], \\
N_{A}(2)=\left[\begin{array}{ll}
0.13 & 0.23
\end{array}\right], \quad G_{A}(2)=[0.5] \\
W(2)=\left[\begin{array}{ll}
0.2 & 0.0 \\
0.0 & 0.2
\end{array}\right], \\
B(2)=\left[\begin{array}{ll}
1.0 & 0.0 \\
0.0 & 1.0
\end{array}\right], \quad M_{B}(2)=\left[\begin{array}{l}
0.12 \\
0.15
\end{array}\right] \\
N_{B}(2)=\left[\begin{array}{ll}
0.13 & 0.23
\end{array}\right], \quad G_{B}(2)=[0.5] \\
C(2)=\left[\begin{array}{ll}
1.0 & 0.0 \\
0.0 & 1.0
\end{array}\right], \quad M_{C}(2)=\left[\begin{array}{l}
0.12 \\
0.15
\end{array}\right] \\
N_{C}(2)=\left[\begin{array}{ll}
0.13 & 0.23
\end{array}\right], \quad G_{C}(2)=[0.5] .
\end{gathered}
$$

The transition probability rate matrix between the two modes is given by

$$
\Lambda=\left[\begin{array}{cc}
-2.0 & 2.0 \\
3.0 & -3.0
\end{array}\right]
$$

Let $\varepsilon_{A}(1)=\varepsilon_{A}(2)=0.5, \varepsilon_{B}(1)=\varepsilon_{B}(2)=0.1$ and $\varepsilon_{C}(1)=$ $\varepsilon_{C}(2)=0.1$; in view of inequalities (39)-(41), we have

$$
\begin{aligned}
X(1) & =\left[\begin{array}{cc}
5.0423 & -0.2399 \\
-0.2399 & 2.9182
\end{array}\right], \\
X(2) & =\left[\begin{array}{ll}
4.8461 & 0.1559 \\
0.1559 & 4.4064
\end{array}\right], \\
Y(1) & =\left[\begin{array}{cc}
0.5467 & -0.7160 \\
-0.7160 & 1.3711
\end{array}\right], \\
Y(2) & =\left[\begin{array}{cc}
1.0892 & -0.7109 \\
-0.7109 & 0.6812
\end{array}\right], \\
\mathbb{k}_{B}(1) & =\left[\begin{array}{cc}
-6.8119 & -0.4532 \\
-0.4532 & -8.6839
\end{array}\right], \\
\mathbb{k}_{B}(2) & =\left[\begin{array}{cc}
-4.1475 & -2.0622 \\
-2.0622 & -4.1055
\end{array}\right], \\
\mathbb{k}_{C}(1) & =\left[\begin{array}{cc}
-1.7326 & 3.2241 \\
3.2241 & -6.7326
\end{array}\right], \\
\mathbb{k}_{C}(2) & =\left[\begin{array}{cc}
-5.1742 & 2.7434 \\
2.7434 & -1.5899
\end{array}\right] .
\end{aligned}
$$

Then according to Theorem 6, the dynamic output feedback controller gains are obtained as follows:

$$
\begin{aligned}
& K_{A}(1)=\left[\begin{array}{cc}
1.8030 & 4.3860 \\
4.3860 & -4.1137
\end{array}\right], \\
& K_{B}(1)=\left[\begin{array}{cc}
0.5083 & -2.5782 \\
-1.9725 & 0.4493
\end{array}\right], \\
& K_{C}(1)=\left[\begin{array}{cc}
-0.2829 & 2.2038 \\
-1.6886 & -5.7923
\end{array}\right], \\
& K_{A}(2)=\left[\begin{array}{cc}
-4.8295 & 2.0890 \\
6.7584 & 6.3166
\end{array}\right], \\
& K_{B}(2)=\left[\begin{array}{cc}
-0.5943 & -1.3272 \\
-1.8660 & -1.6399
\end{array}\right], \\
& K_{C}(2)=\left[\begin{array}{cc}
-6.6548 & -2.9176 \\
3.1218 & 0.9240
\end{array}\right] .
\end{aligned}
$$

Therefore, the designed dynamic output feedback controller (38) is feasible and guarantees the stochastic stability of the Itô stochastic uncertain MJLS in (36).

\section{Conclusions}

The robustly stochastic stabilization problem for the Markov jumping Itô stochastic linear systems with linear fractional uncertainties had been investigated via state feedback and dynamic output feedback. Sufficient conditions, by solving linear matrix inequalities, are presented to realize the design of controller. The robust $H_{\infty}$ control problem via state feedback and dynamic output feedback for such Itô stochastic MJLS with linear fractional uncertainties should be studied in future work. 


\section{Acknowledgments}

The authors also gratefully acknowledge the helpful comments and suggestions of the reviewers, which have improved the presentation. This work was partially supported by the Natural Science Foundation of China under Grant (nos. 61263005 and 61065010), the Research Fund for the Doctoral Program of Higher Education under Grant no. 20105201120003, the Program for New Century Excellent Talents in University, and the Graduate School Innovation Foundation of Guizhou University.

\section{References}

[1] Z. Wu, P. Shi, H. Su, and J. Chu, "Passivity analysis for discretetime stochastic Markovian jump neural networks with mixed time delays," IEEE Transactions on Neural Networks, vol. 22, no. 10, pp. 1566-1575, 2011.

[2] Y. Fang and K. A. Loparo, "Stochastic stability of jump linear systems," IEEE Transactions on Automatic Control, vol. 47, no. 7, pp. 1204-1208, 2002.

[3] J. Xiong and J. Lam, "Stabilization of discrete-time Markovian jump linear systems via time-delayed controllers," Automatica, vol. 42, no. 5, pp. 747-753, 2006.

[4] L. Zhang and J. Lam, "Necessary and sufficient conditions for analysis and synthesis of markov jump linear systems with incomplete transition descriptions," IEEE Transactions on Automatic Control, vol. 55, no. 7, pp. 1695-1701, 2010.

[5] D. Yue, J. Fang, and S. Won, "Delay-dependent robust stability of stochastic uncertain systems with time delay and markovian jump parameters," Circuits, Systems, and Signal Processing, vol. 22, no. 4, pp. 351-365, 2003.

[6] P. Bolzern, P. Colaneri, and G. De Nicolao, "Markov Jump Linear Systems with switching transition rates: mean square stability with dwell-time," Automatica, vol. 46, no. 6, pp. 10811088, 2010.

[7] J. Wang and H. Yang, "Output-feedback stabilization of Markovian jump linear systems over networks with time delays," Journal of Information and Computational Science, vol. 8, no. 13, pp. 2545-2552, 2011.

[8] N. Xiao, L. Xie, and M. Fu, "Stabilization of Markov jump linear systems using quantized state feedback," Automatica, vol. 46, no. 10, pp. 1696-1702, 2010.

[9] H. Zhang, Y. Shi, and A. Mehr, "Robust static output feedback control and remote PID design for networked motor systems," IEEE Transactions on Industrial Electronics, vol. 58, no. 12, pp. 5396-5405, 2011

[10] Z. Shu, J. Lam, and J. Xiong, "Non-fragile exponential stability assignment of discrete-time linear systems with missing data in actuators," IEEE Transactions on Automatic Control, vol. 54, no. 3, pp. 625-630, 2009.

[11] L. Ma and F. Da, "Exponential $H_{\infty}$ filter design for stochastic time-varying delay systems with Markovian jumping parameters," International Journal of Robust and Nonlinear Control, vol. 20, no. 7, pp. 802-817, 2010.

[12] W. Chen, Q. Ma, G. Miao, and Y. Zhang, "Stability analysis of stochastic neural networks with Markovian jump parameters using delay-partitioning approach," Neurocomputing, vol. 103, pp. 22-28, 2013.

[13] S. Wen, Z. Zeng, and T. Huang, "Reliable Ho filter design for a class of mixed-delay markovian jump systems with stochastic nonlinearities and multiplicative noises via delay-partitioning method," International Journal of Control, Automation and Systems, vol. 10, no. 4, pp. 711-720, 2012.

[14] S. Sathananthan, C. Beane, G. S. Ladde, and L. H. Keel, "Stabilization of stochastic systems under Markovian switching," Nonlinear Analysis: Hybrid Systems, vol. 4, no. 4, pp. 804-817, 2010.

[15] C. Yuan and J. Lygeros, "Stabilization of a class of stochastic differential equations with Markovian switching," Systems and Control Letters, vol. 54, no. 9, pp. 819-833, 2005.

[16] C. Yuan and X. Mao, "Robust stability and controllability of stochastic differential delay equations with Markovian switching," Automatica, vol. 40, no. 3, pp. 343-354, 2004.

[17] Y. Niu, D. W. C. Ho, and X. Wang, "Sliding mode control for Itô stochastic systems with Markovian switching," Automatica, vol. 43, no. 10, pp. 1784-1790, 2007.

[18] E. K. Boukas, Stochastic Switching Systems: Analysis and Design, Birkhauser, Boston, Mass, USA, 2005.

[19] F. Long, S. Fei, Z. Fu, S. Zheng, and W. Wei, " $H_{\infty}$ control and quadratic stabilization of switched linear systems with linear fractional uncertainties via output feedback," Nonlinear Analysis: Hybrid Systems, vol. 2, no. 1, pp. 18-27, 2008.

[20] L. Xie, "Output feedback $H_{\infty}$ control of systems with parameter uncertainty," International Journal of Control, vol. 63, no. 4, pp. 741-750, 1996. 

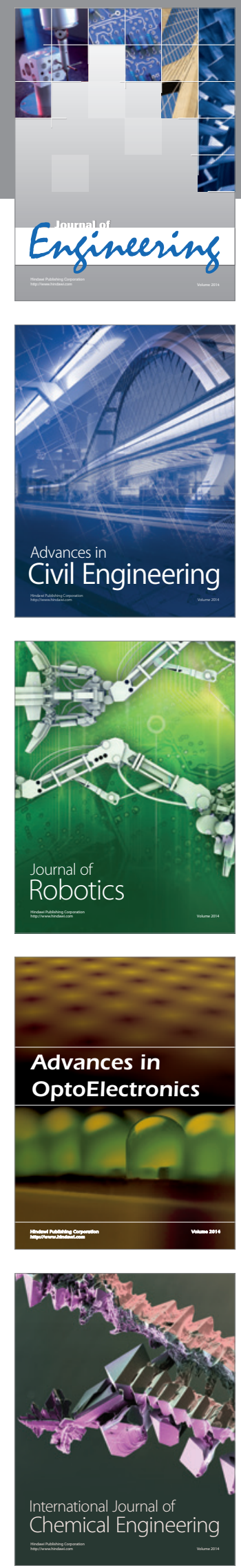

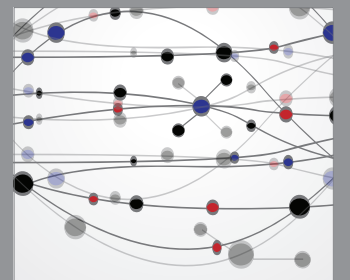

The Scientific World Journal
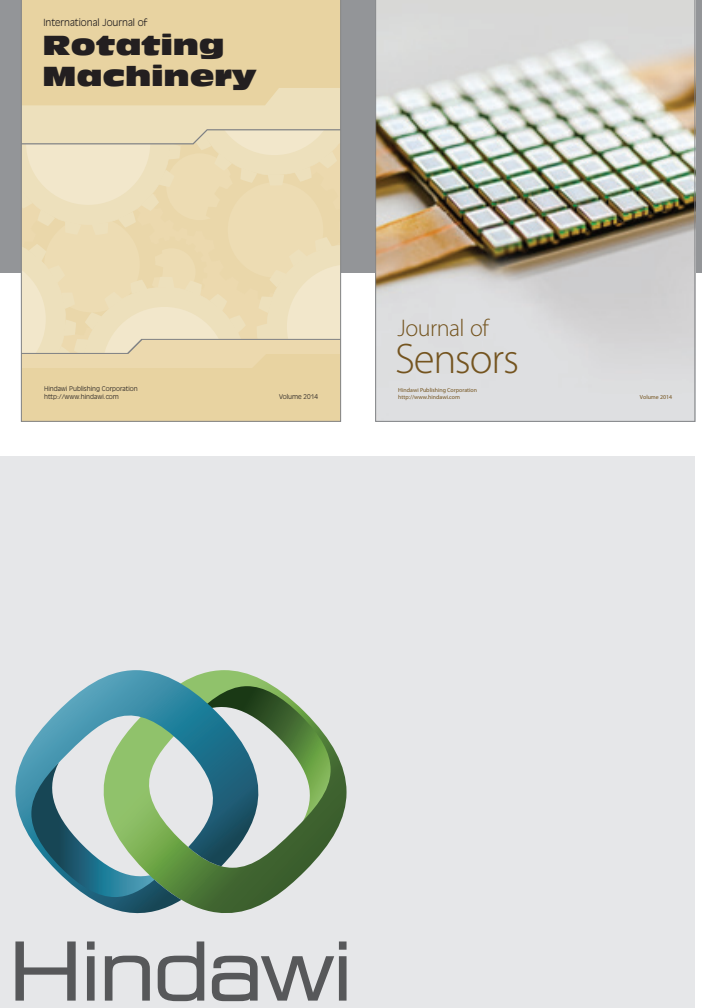

Submit your manuscripts at http://www.hindawi.com
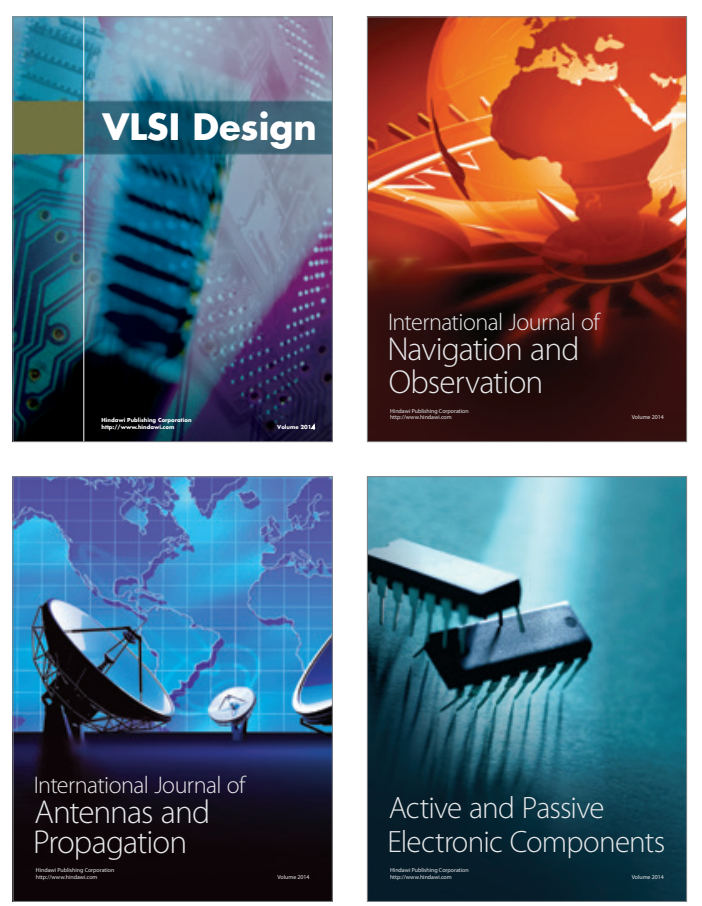
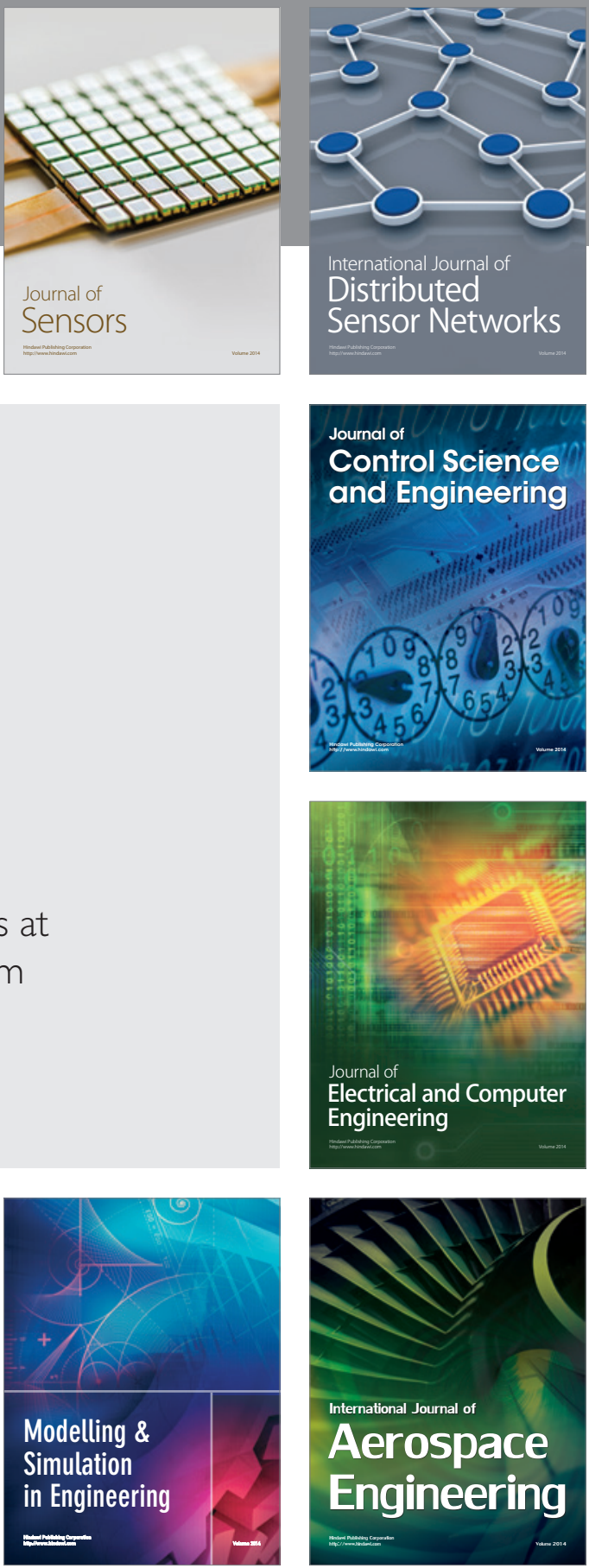

Journal of

Control Science

and Engineering
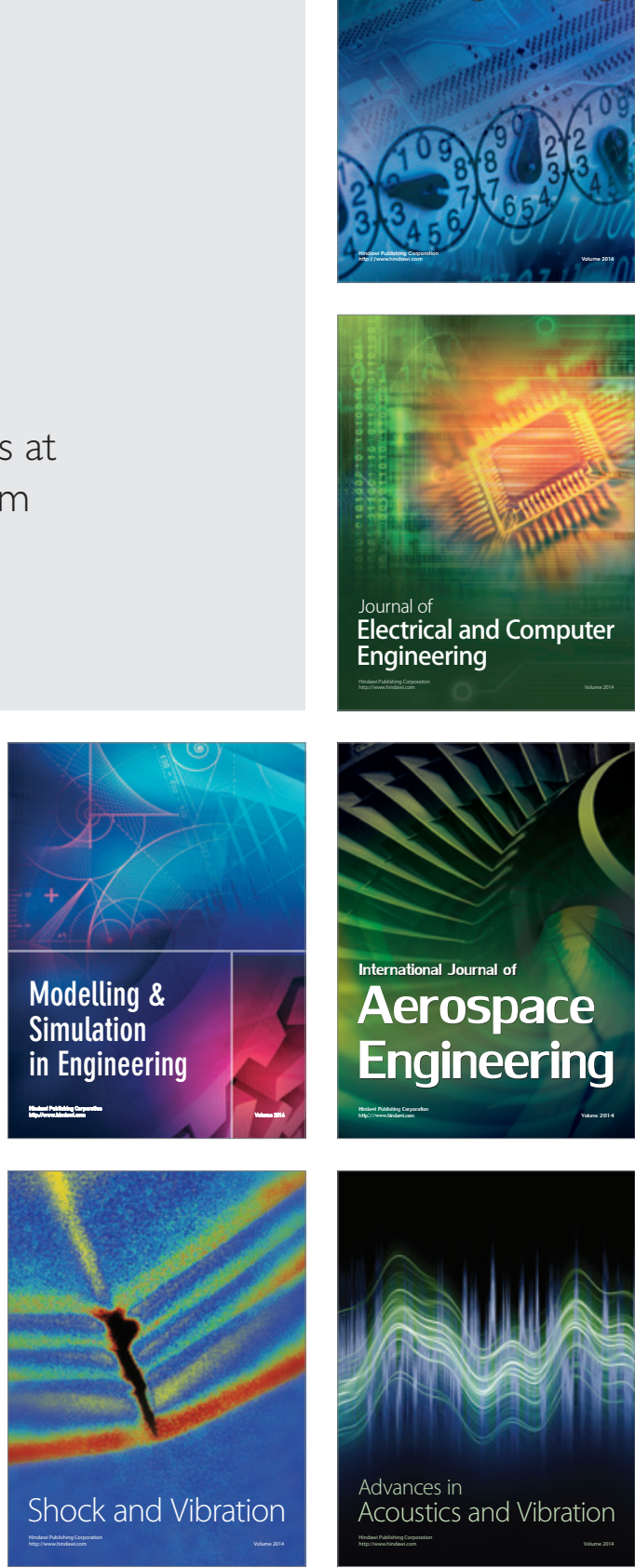